\title{
Influence of Preparation Method on the Structural, Linear and Nonlinear Optical Properties of TiN Nanoparticles
}

Mahdi Safa

Damghan University

Yasser Rajabi ( $\nabla$ y.rajabi@du.ac.ir)

Damghan university https://orcid.org/0000-0002-0272-7305

Mahdi Ardyanian

Damghan University

\section{Research Article}

Keywords: Influence of Preparation, TiN Nanoparticles, Nonlinear Optical, saturation absorption

Posted Date: February 25th, 2021

DOI: https://doi.org/10.21203/rs.3.rs-241533/v1

License: (c) (i) This work is licensed under a Creative Commons Attribution 4.0 International License.

Read Full License 


\title{
Influence of preparation method on the structural, linear and nonlinear optical properties of TiN nanoparticles
}

\author{
Mahdi Safa • Yasser Rajabi* . Mahdi \\ Ardyanian
}

Received: date / Accepted: date

\begin{abstract}
In this paper, titanium nitride nanoparticles were prepared in three methods, In the first method, titanium nitride nanoparticles were prepared by the Ball-mill method; in the second and third methods, the nanoparticles were synthesized by the sol-gel, and the co-precipitation methods. The samples were characterized by X-ray diffraction (XRD), UV-Vis spectroscopy, and scanning electron microscopy (SEM), for the study of structural, linear optical properties, and surface morphology study, respectively. The Z-scan technique was utilized to study the TiN nanoparticles nonlinearity. A change in nonlinear optical behavior with an increase in input pump power and concentration of the TiN nanoparticles was observed. Results showed that the TiN nanoparticles give new potentials in nonlinear optical applications. The nonlinear optical behavior of the TiN nanoparticles shifts from inverse saturation absorption to saturation absorption with increase of intensity.
\end{abstract}

\section{Introduction}

Light control by changing the optical functions leading to the control and manipulation of the light - matter Interactions, plays an important and key role in the design and development of optical devices [1-4]. In fact, with this achievement, a change of the optical parameters is possible, which is an interesting fundamental scientific tool that leads to many advances in technologies

\footnotetext{
M. Safa

School of Physics, Damghan University, Damghan, 36716-41167, Semnann, Iran.

E-mail: mm.merlin.safa@gmail.com

Y. Rajabi (* Corresponding author)

School of Physics, Damghan University, Damghan, 36716-41167, Semnann, Iran.

E-mail: y.rajabi@du.ac.ir

M. Ardyanian

School of Physics, Damghan University, Damghan, 36716-41167, Semnann, Iran.

E-mail: ardyanian@du.ac.ir
} 
related to optics and lasers [5-8]. Due to the great interest of researchers in this area, the research in the nonlinear optics field has grown significantly [1-4,9-11]. One way to achieve this goal is to make materials with different synthesis methods [12-14]. It is also possible to prepare the material in different concentrations and study its nonlinear behavior in different conditions $[15,16]$. Therefore, the preparation of such materials will improve the optical properties. These materials can replace common metals in optical applications. Nanoscience is becoming increasingly important as a pioneering technology in the production of such materials. These materials have been considered by many researchers due to their use for dual optical and mechanical applications. Among a wide range of these materials, metal nitrides are known as inter-metallic materials due to their electronic structure similarity to metals. Titanium nitride $(T i N)$ is an example of such a material[1721]. Several outstanding features such as high hardness properties (2000 kg $/ \mathrm{mm}^{2}$ ), corrosion resistance and high melting point (2930 degrees Celsius), good compatibility with the body, different production methods, and low manufacturing costs, make it a suitable alternative to commonly used metals (gold and silver) in optical applications. Proper electrical conductivity has made it a special electrical ceramic for self-heating plants and a conductor for electronic applications. The properties of titanium nitride generally depend on the stoichiometry (titanium to nitrogen molar ratio), impurities (such as oxygen and chloride), and the structure that can be controlled by changing the synthesis method and process parameters [22-26]. Various methods have been reported to synthesize of titanium nitride in bulk form or thin films using the solid-state method[27-37]. These methods included chemical vapor deposition (CVD)[28] and physical vapor deposition (PVD) [27,29], pulsed laser deposition (PLD)[30], sputtering[27], and chemical methods such as sol-gel[33], and evacuation of electric arc. Most of these methods are performed at high temperatures and high pressures and often require a lot of equipment. Titanium nitride is an intermetallic material with a dark-blue color spectrum. It has different phases; the main phase of $T i N$ is in the form of face-centered cubic structure (FCC), with a lattice parameter of 4.24 angstroms.

In addition to mechanical and chemical properties, the optical properties of this compound are so important. One of the optical applications of $T i N$ is its utilization in anti-reflective coatings and optical filters [25]. In medical science, it is used to increase quality and characteristics such as lightness, high strength, less abrasion and more trenchancy of medical instruments. $T i N$ is also used in pigmented solar cells for increasing light absorption and solar cell efficiency [38-41]. Experimental results have shown that performance of $T i N$ is more efficient than gold at high temperatures and light intensities. Therefore, the use of $T i N$ can be of great interest in nonlinear optics applications [43-45]. Assistance in photonic development, spectroscopy, fiber optic lines, optical switches, etc., requires knowing the nonlinear optical behavior of materials. Many of the nonlinear optics phenomena result from nonlinear refractive index and nonlinear absorption coefficient. The research community has been looking for nonlinear materials to develop and manufacture light-controllable 

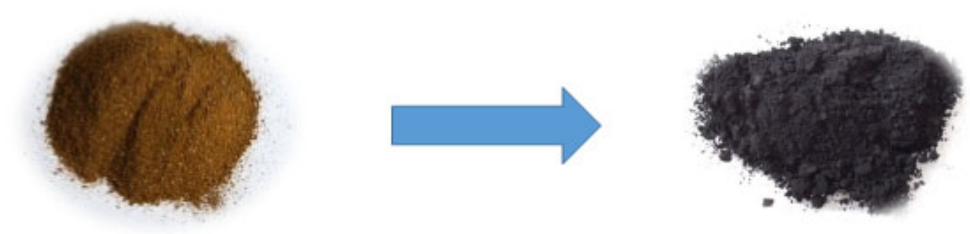

Fig. 1 Left: Sample before Ball mill process, Right: resulted sample after Ball mill process.

materials. There are many methods for measuring optical parameters accurately. One of the best ways for simultaneous the nonlinear refractive index and the nonlinear absorption coefficient is the Z-scan method [46-49].

In this paper, titanium nitride was synthesized in different ways to achieve the goal of controlling the interaction of light and matter. Preparation of TiN nanoparticles was performed using simple methods at low temperature, and atmospheric pressure. Then, from each sample, different concentrations were prepared, and the nonlinear optics behavior of $T i N$ was studied. The nonlinear interaction of a continuous wave (CW) diode-pumped second harmonic NdYag laser with a power of $150 \mathrm{~mW}$ at $532 \mathrm{~nm}$ was studied using open-aperture and close aperture Z-scan technique. This setup was used to measure the nonlinear refractive index and nonlinear absorption coefficient variation of TiN nanoparticles. Also, the nonlinear behavior of the prepared samples at different laser intensities was studied.

\section{Materials}

Titanium isopropoxide, hydrazine, acetonitrile, ethanol, ethylene glycol Washing the tools Before starting the test, all used equipment was washed with ethanol, and then with acetone and dried at $120^{\circ} \mathrm{C}$.

\section{Preparation}

In this paper, titanium nitride was prepared by both physical and chemical methods. The Ball mill method was used as a physical process, and two chemical processes were used as co-precipitation and sol-gel methods.

\subsection{Ball-mill process}

To prepare titanium nitride nanoparticles, samples were purchased from Merck company with a particle size of up to 300 micrometers. The left Figure 1 shows the primary sample. Then, a Ball mill was used to grind and pulverize the powder, and $10 \mathrm{~g}$ of $T i N$ particles were poured into the chamber. After checking the device settings, milling was done. Samples were placed in this process for 30 minutes at 350 and $500 \mathrm{rpm}$ rotation rate of the balls. At the 


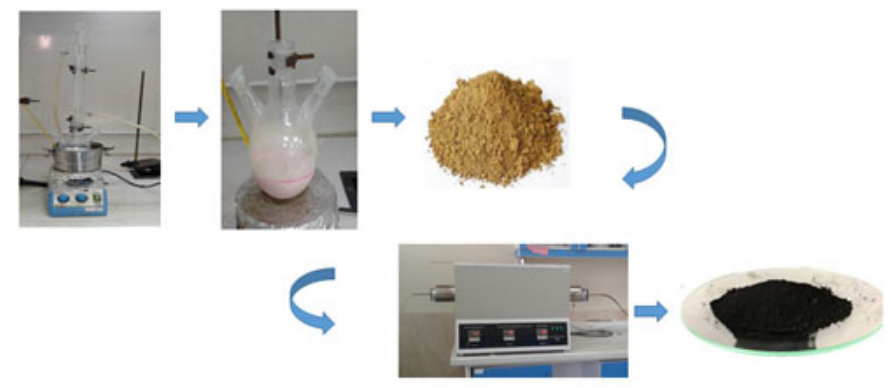

Fig. 2 The preparation process of TiN by co-precipitation method.

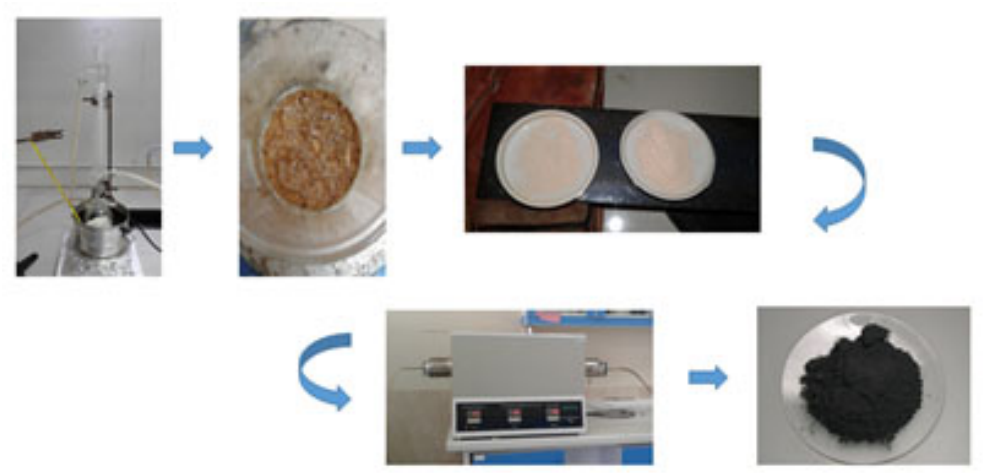

Fig. 3 The preparation process of TiN by sol-gel method.

end of the mill, the nanoparticles are removed from the chamber, shown in the right Figure 1.

\subsection{Co-precipitation method}

Firstly, for preparing titanium nitride by co-precipitation method, needed equipments were washed with deionized water and acetone, thoroughly, and then dried at $120^{\circ} \mathrm{C}$. The $0.339 \mathrm{M}$ clear solution of Titanium isopropoxide (TTIP) was prepared after adding the acetonitrile, stirring duting $20 \mathrm{~min}$ utes at $80^{\circ} \mathrm{C}$. Then hydrazine was added to solution drop-wise to change its colour to creamy (light brown). The solution was refluxed for 12 hours in an oil bath at a uniform temperature of $80^{\circ} \mathrm{C}$. Finally, using a vacuum desiccator, the solution was kept at $120^{\circ} \mathrm{C}$ for 15 hours and then at room temperature for 5 hours. The resulting dry powder had a brown color. The resulting powder was exposed to ammonia gas at $1000^{\circ} \mathrm{C}$ and $500 \mathrm{SCCM}$ gas flow for 5 hours, and finally dark brown titanium nitride nanoparticles were obtained. Figure 2 shows the steps of $T i N$ synthesis by co-precipitation method. 


\subsection{Sol-gel method}

For synthesis of $T i N$ by sol-gel method, Titanium isoperoxide (TTIP) was immersed in nitrogen gas for 10 minutes; after 20 minutes stirring at $80^{\circ} \mathrm{C}$, ethanol was added to solution slowl and ethylene glycol was added drop-wise as a complexing agent. The sol was obtained after 9 hours reflux in oil bath at $80^{\circ} \mathrm{C}$; then the gel was obtained after heating and drying at $120^{\circ} \mathrm{C}$ for 15 hours in the air and and 3 hours in vacuum respectively. The gel was prepared as powder, and finally $T i N$ nanoparticles were obtained after placement of the powder under ammonia exposure for 5 hours at 500 SCCM.

Figure 3 shows the steps of performing the test.

\section{Characterization methods}

The study of the structure, linear optics properties, and morphology of the samples, ware performed by x-ray diffraction, Uv-Vis spectroscopy, and scanning electron microscopy (SEM), Respectively. Moreover, the samples chemical bonds were also investigated via Fourier transform infrared spectroscopy.

\subsection{Nonlinear optical setup}

The nonlinear optical properties, the nonlinear refractive index, $n_{2}$, and the nonlinear absorption coefficient, $\beta$, of the samples were investigated experimentally by the Z-scan technique [46-49]. The Z-scan setup is shown in the Figure 4.

The Nd-Yag C. W. laser beam with Gaussian intensity distribution, the wavelength of $532 \mathrm{~nm}$ was performed. The laser beam power was $150 \mathrm{~mW}$ with a beam waist of $6 \mu \mathrm{m}$. The intensity of the laser beam was controlled by the neutral density filters. The laser beam converged by a lens with $\mathrm{f}=15 \mathrm{~cm}$. The sample moves along the axis of the light beam, near the focal point. Two intensity detectors (Thorlabs, model PM100) are used to investigating the nonlinear refractive index and nonlinear absorption coefficient. The first intensity detector was used to measure the nonlinear refractive index, $n_{2}$, and the second intensity detector was used to measure the nonlinear absorption coefficient, $\beta$. In this experiment, first, the intensity of the input light beam was measured without the presence of the sample. Then the different samples (TiN nanoparticles), with varying concentrations within a $1 \mathrm{~mm}$ thickness quartz cell, were moved by a high-precision optical rail. The samples were displaced along the z-direction of the beam (through the focus of the lens). The optical rail was accurately shifted along the z-direction near the focal point of the lens using a DC stepper motors, automatically. The variations of intensity corresponding to the different positions of the sample were recorded by two intensity detector(Model PM100) for open and close aperture; these variations were, then, stored in a computer automatically. 


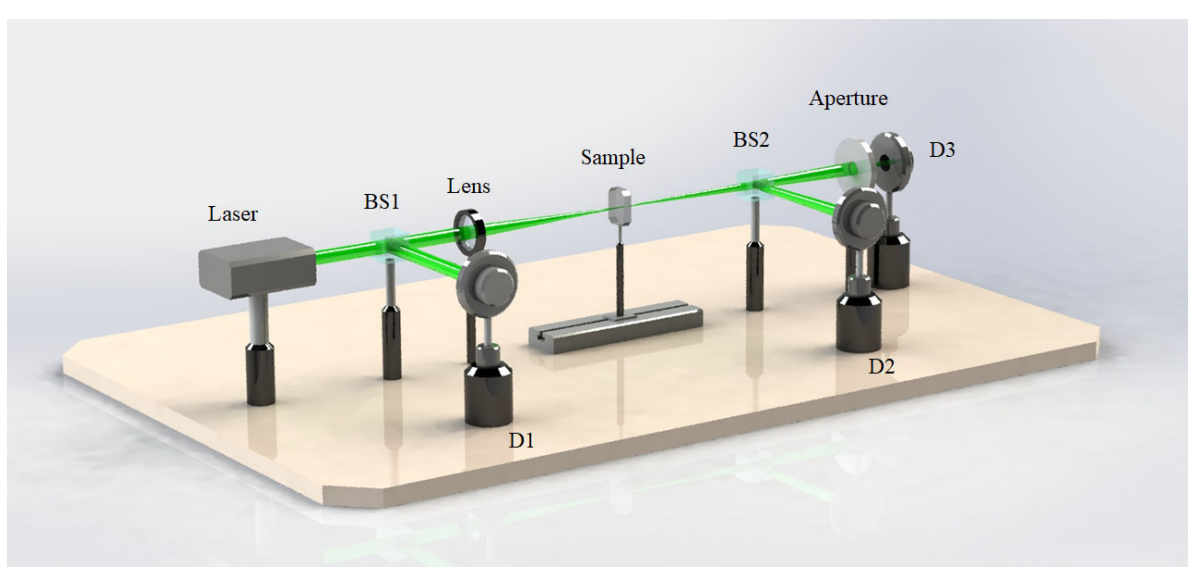

Fig. 4 The experimental setup of open-aperture and close-aperture the Z-scan technique: Laser is Nd-Yag, $D_{1}, D_{2}$ and $D_{3}$ are intensity detectors, BS1 and BS2 are beam splitter.

\section{Results and discussions}

\subsection{Structural study}

\subsubsection{Ball Mill method}

Figure 5a represents a comparison of the reference XRD diffraction pattern (down) and the samples prepared by the Ball mill method. The reference XRD pattern corresponds to the TiN powder purchased from Merck. The strong peaks along [111], [200], [220], [311], and [222] crystallographic directions are well observed at $36.76^{\circ}, 42.56^{\circ}, 61.76^{\circ}, 74.12^{\circ}$, and $77.96^{\circ}$ respectively with the lattice constant of $4.241 A^{\circ}$ (JCPDS card No.1420-38). Two upper graphs represent the samples prepared by the Ball mill method. The patterns show that the milling process creates a peak at an angle of $43.64^{\circ}$ corresponding to the (210) plane of $\mathrm{TiO}_{2}$ Rutile phase (JCPDS 88-1175) that is shown as an impurity on the graph with a+ sign. This oxide phase could be due to the warming and oxidation of titanium during the milling process. The intensity of this peak is higher in the sample prepared at a lower rotation rate (350 $\mathrm{rpm})$. By increasing the number of rotations, the rotation rate, and time of the milling process, the intensity of the $\mathrm{TiN}$ peaks increased, and the $\mathrm{TiO}_{2}$ peak at $43.64^{\circ}$ decreased. A fine shift of the peaks to higher angles in the milled samples (see table 1) could be attributed to a decrease of Bragge planes distances due to fabricating of nanoparticles. The nanocrystallites size are estimated by Scherrer equation [49]:

$$
D=\frac{k \lambda}{\beta \operatorname{Cos} \theta}
$$


where $\mathrm{k}$ is a constant, which is about 0.89 for spherical nanoparticles, $\lambda$ is the wavelength of $C u k \alpha$ peak equal $0.15406 \mathrm{~nm}$. $\theta$ is the Bragge diffraction angle. $\beta$ is the FWHM of the peak.

\subsubsection{Sol-gel method}

Figure 5b represents the XRD patterns of the samples synthesized by the solgel method followed by calcining in ammonia atmosphere between $700-1000^{\circ} \mathrm{C}$. As presented, as prepared sample presents the pattern of rutile phase of $\mathrm{TiO}_{2}$. The effect of calcination temperature is indicated in upper patterns. It is observed that after calcining, the rutile phase of $\mathrm{TiO}_{2}$ evolved to $\mathrm{TiN}$. At $700^{\circ} \mathrm{C}$ there is a binary system of Brokite phase of $\mathrm{TiO}_{2}$ (denoted by +)(JCPDS card No. 29-1360) and $\operatorname{TiN}$ (denoted by *) (JCPDS card No.1420-38). At T $=800^{\circ} \mathrm{C}$ the structure has changed to $T i N$ with some weak diffraction peaks which are disappeared after calcining at $\mathrm{T}=1000^{\circ} \mathrm{C}$, the sample is completely purified without titanium oxide phases containing main crystallographic directions of [111], [200], [220], [311], and [222] of TiN.

\subsubsection{Co-precipitation method}

Figure 5c shows a sample synthesized by the co-precipitation method. These patterns indicate the effect of calcination of the sample at temperatures of $700-1000{ }^{\circ} \mathrm{C}$ in an ammonia atmosphere. In this sample, with increasing the calcination temperature, an evolution in the structure from $\mathrm{TiO}_{2}$ to $\mathrm{TiN}$ has occurred. At $700^{\circ} C$, titanium oxide dual phase of anatase and the rutile are observed. As the temperature rises to $800^{\circ} \mathrm{C}$, the $\operatorname{TiN}$ phase appeared (denoted by ${ }^{*}$ ) while the $\mathrm{TiO}_{2}$ phase (denoted by + ) still exists. At $\mathrm{T}=1000^{\circ} \mathrm{C}$, the completely purified crystalline the $\mathrm{TiN}$ phase is observed without any $\mathrm{TiO}_{2}$ phase.

\subsection{Uv-visible spectroscopy study}

Figure 6 shows the transmittance curves for titanium nitride nanoparticles. As can be seen, the sample synthesized by the co-precipitation method has the highest, and the sample synthesized by the sol-gel method has the lowest transparency in the visible wavelength range compared to other samples; which could be attributed to smaller nanoparticles sizes in the samples prepared by co-precipitation method. Also, all samples have the same absorption edge, because they have the same structure after calcination at $\mathrm{T}=1000^{\circ} \mathrm{C}$ in ammonia.

\subsection{FT-IR spectroscopy study}

FT-IR spectroscopy was effectuated for investigating the type of chemical bonds in the synthesized samples. Figure 7 a represents the IR transmittance 

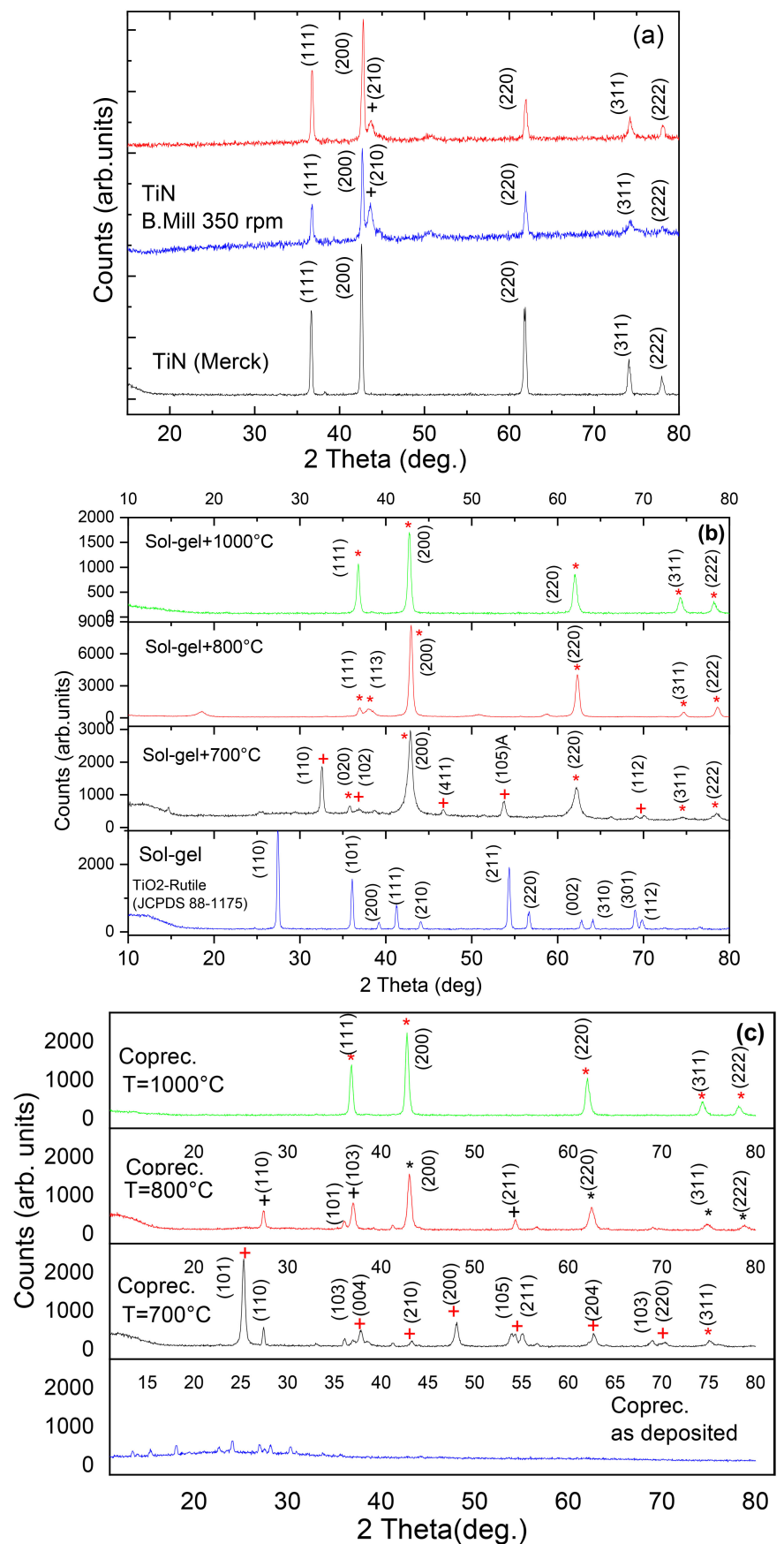

Fig. 5 a - Comparison of the XRD pattern of the sample purchased from Merck, and TiN nanoparticles prepared by Ball. Mill technique. $\mathrm{b}$ - The effect of calcination temperature in ammonia atmosphere on the structure of the samples synthesized by the sol-gel method, $\mathrm{c}-$ XRD pattern of the sample synthesized by co-precipitation calcinated at different temperatures in ammonia atmosphere, 
Table 1 Crystallographic data of TiN prepared in different methods.

\begin{tabular}{|c|c|c|c|c|c|c|}
\hline Samples & hkl & $2 \theta$ & FWHM $\left(\times 10^{-3}\right)$ & $\mathrm{d}(\dot{A})$ & $\mathrm{D}(\dot{A})$ & $\Delta\left(\times 10^{-4}\right)$ \\
\hline \hline \multirow{5}{*}{ Merk } & 111 & 36.653 & 4.79 & 2.449 & 3050 & 10.7 \\
& 200 & 42.587 & 4.95 & 2.121 & 3000 & 1.11 \\
& 220 & 61.839 & 5.68 & 1.499 & 2840 & 12.39 \\
& 311 & 74.086 & 6.83 & 1.278 & 2540 & 15.5 \\
& 222 & 77.965 & 7.01 & 1.224 & 2580 & 15.02 \\
\hline \multirow{5}{*}{ Ball mill(500) } & 111 & 36.721 & 4.12 & 2.447 & 354 & 79.79 \\
& 200 & 42.789 & 3.43 & 2.11 & 434 & 53.09 \\
& 220 & 61.936 & 5.49 & 1.49 & 294 & 11.56 \\
& 311 & 74.193 & 4.80 & 1.278 & 362 & 76.31 \\
& 222 & 78.124 & 8.37 & 1.222 & 213 & 22.04 \\
\hline \multirow{5}{*}{ Ball mill(350) } & 111 & 36.76 & 4.12 & 2.444 & 354 & 79.79 \\
& 200 & 42.68 & 3.43 & 2.118 & 434 & 53.09 \\
& 220 & 61.931 & 2.74 & 1.498 & 590 & 28.72 \\
& 311 & 74.23 & 8.23 & 1.227 & 211 & 22.46 \\
& 222 & 78.039 & 10.04 & 1.223 & 177 & 31.91 \\
\hline \multirow{5}{*}{ Co-precipitated } & 111 & 36.83 & 5.49 & 2.44 & 267 & 14.02 \\
& 200 & 42.746 & 5.49 & 2.1153 & 271 & 13.61 \\
& 220 & 62.061 & 5.49 & 1.495 & 294 & 11.56 \\
& 311 & 74.35 & 3.43 & 1.2758 & 507 & 38.9 \\
& 222 & 78.129 & 5.86 & 1.223 & 304 & 10.82 \\
\hline & 111 & 36.776 & 4.8 & 2.4438 & 304 & 10.82 \\
& 200 & 42.7977 & 5.49 & 2.1129 & 271 & 13.61 \\
& 220 & 62.031 & 3.43 & 1.4961 & 471 & 45.07 \\
& 311 & 74.35 & 4.12 & 1.2758 & 422 & 56.15 \\
& 222 & 78.159 & 5.861 & 1.2219 & 304 & 61.26 \\
\hline
\end{tabular}

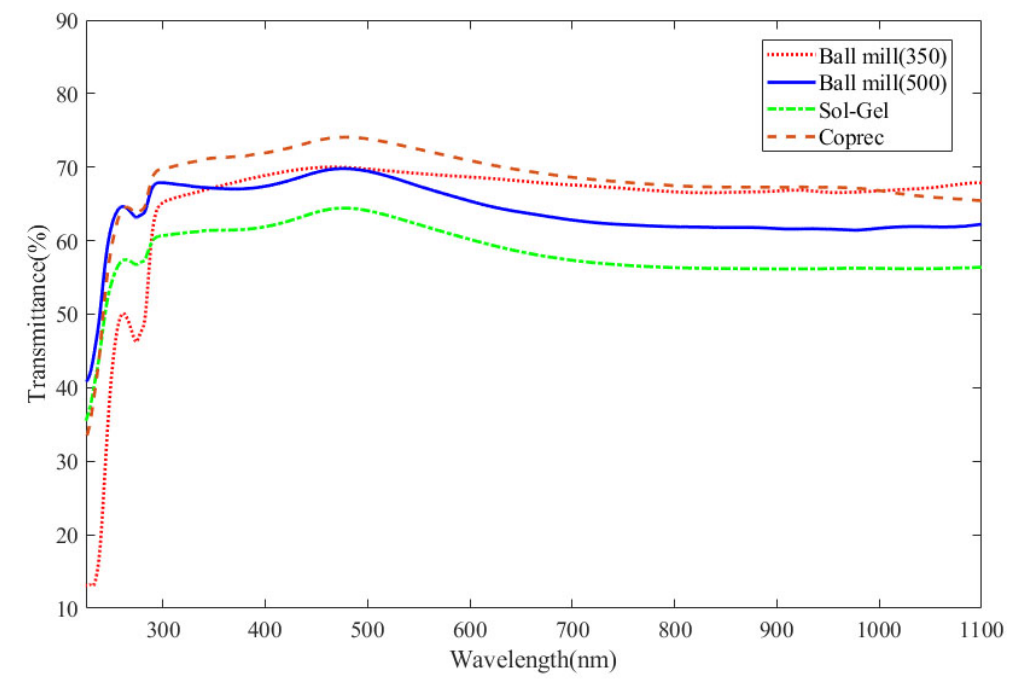

Fig. $6 \mathrm{UV}-\mathrm{V}$ is spectra of $\mathrm{TiN}$. 
(a)

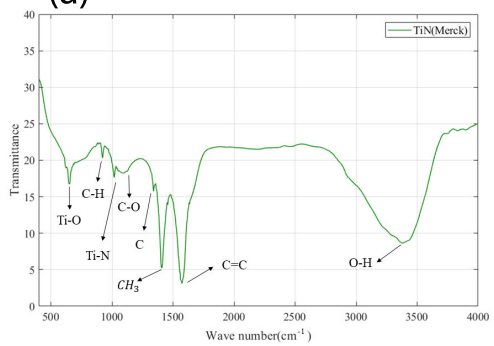

(b)

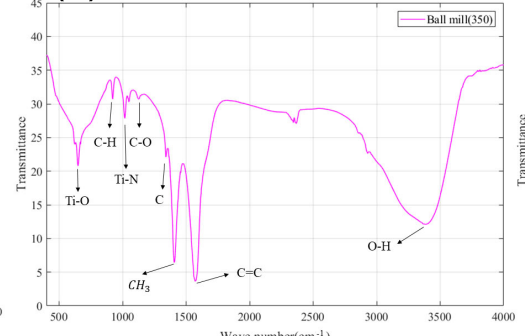

(c)

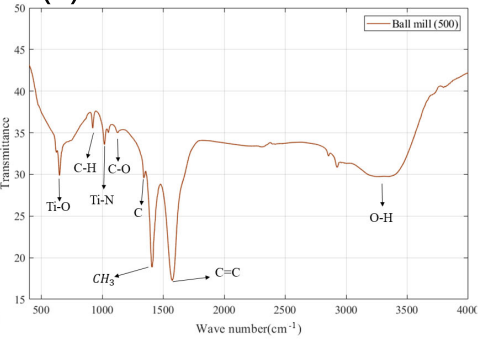

(d)
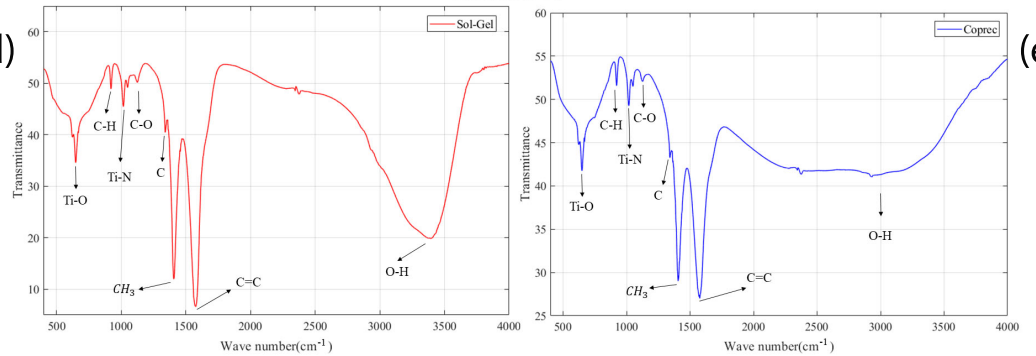

(e)

Fig. 7 FTIR spectra of sample prepared by a: Merck, b: the Ball mill method with 350rpm, c: the Ball mill method with 500rpm, d: the sol-gel method, e: the co-precipitation method.

of the Titanium nitride sample purchased from Merck Company, Figure 7b represents the Ball milled sample by $350 \mathrm{rpm}$ rotation rate, and Figure $7 \mathrm{c}$ corresponds to the same sample at $500 \mathrm{rpm}$. Figure $7 \mathrm{~d}$ Sample also represents the synthesized sample by sol-gel method, and Figure 7e corresponds to the sample synthesized by the co-precipitation method.

In all of the spectra, the absorbance in the range of $3200 \mathrm{~cm}^{-1}$ to $3700 \mathrm{~cm}^{-1}$, corresponds to $O-H$ bond due to the presence of water and alcohol solvents in the sample remained from synthesis process. In the range of $1570 \mathrm{~cm}^{-1}$, a double carbon bond is observed as $C=C$, which is due to carbonyl bonds. Absorbance at $1406 \mathrm{~cm}^{-1}$, is related to the $C-H_{3}$ bond chain in the samples. At $1343 \mathrm{~cm}^{-1}$, a weak absorbance attributed to $C$ bond is observed, which is a sign of a unique carbon bond. The absorbance around $1122 \mathrm{~cm}^{-1}$ is related to $C-O$ bond, corresponding to carbon dioxide in the chamber atmosphere. Moreover it could be attributed to carbon and oxygen bond due to epoxy bond. Absorbance at $1017 \mathrm{~cm}^{-1}$, corresponds to $T i-N$ bond, which confirms the formation of a titanium-nitride bond in the process. In the range of $649 \mathrm{~cm}^{-1}$, $T i-O$ bonds show oxygen and titanium bonds [31,50]. As presented, all of the synthesized contain the $T i-N$ bonds confirming the synthesis of TiN after calcining in ammonia atmosphere.

\subsection{Study of surface morphology of titanium nitride nanoparticles}

Scanning Electron Microscope (SEM) images of reference and synthesized samples by different methods are represented in figure 8 . Figure 8 a shows the sur- 
face morphology of the TiN particles perchased from Merck. The images show the particles with homogeneous size, polyhedron, and irregular morphology.

Figure $8 \mathrm{~b}$ shows describes the particles prepared by ball mill method at $350 \mathrm{rpm}$. It is observed pseudo-spheric and aggregated grains with decreased size. The particles size are non-uniform and irregularly multifaceted.

Figure 8c shows the SEM images of the particles prepared by a the Ball mill at $500 \mathrm{rpm}$. The particles are non-uniform and irregularly multifaceted. SEM images of the Ball mill processed samples (Figure 8b and 8c) revealed the porous structure with an average particles size around $100 \mathrm{~nm}$ with an increase in porousity after processing.

Figure $8 \mathrm{~d}$ shows the sample synthesized by the sol-gel method annealed at $1000^{\circ} \mathrm{C}$. The surface morphology shows the more aggregated particles with higher size than ball mill method, while the porousity was decreased.

Figure $8 \mathrm{e}$ shows the images of the sample synthesized by the co-precipitation method annealed at $1000^{\circ} \mathrm{C}$ in amonia atmosphere. Spherical aggregated particles in ball shaped grains with a size up to $100 \mathrm{~nm}$ and smaller than other samples.

\subsection{Determination of the band gap}

For determining the optical band gap of the samples, the curves of the variation of $(\alpha h \nu)^{2}$ versus photon energy, $h \nu$, are represented in Figure 9. The band gap could be determined by extrapolating the straight portion to the energy axis, i.e. $(\alpha h \nu)^{2}=0$ [51]. The band gap of the titanium nitride is reported as direct and equal to $4.3 \mathrm{eV}[52]$. Based on the absorption edges, the prepared samples have two band gaps. Depending on the method of preparation of nanoparticles, their first band gap is from about $1.58 \mathrm{eV}$ for the Ball mill method with 350 rpm to $1.95 \mathrm{eV}$ for the Ball mill processed sample at $500 \mathrm{rpm}$. The second band gap of these samples varies from about $3.25 \mathrm{eV}$ for the Ball mill sample at 500 rpm to about $3.94 \mathrm{eV}$ for the Ball mill sample at $350 \mathrm{rpm}$ (Figure 9).

For the samples synthesized by the co-precipitation method, according to the absorption edges (Figure 5) and Figure 9c, the band gap was estimated to be about $1.75 \mathrm{eV}$ and $3.4 \mathrm{eV}$.

For the samples synthesized by the sol-gel method, according to the absorption edges (Figure 9d), the band gap was estimated to be about $1.82 \mathrm{eV}$ and $3.15 \mathrm{eV}$.

The first band gap can be attributed to the presence of the titanium oxide phase in all of the samples as confirmed by the FT-IR spectroscopy, and the second one corresponds to TiN phase. A band gap tuning has been down in $\mathrm{TiO}_{2}-\mathrm{TiN}$ nanocomposites in the range of $1.68-3.23 \mathrm{eV}$, which are the values near the energies obtained in Figure 9 [53]. 


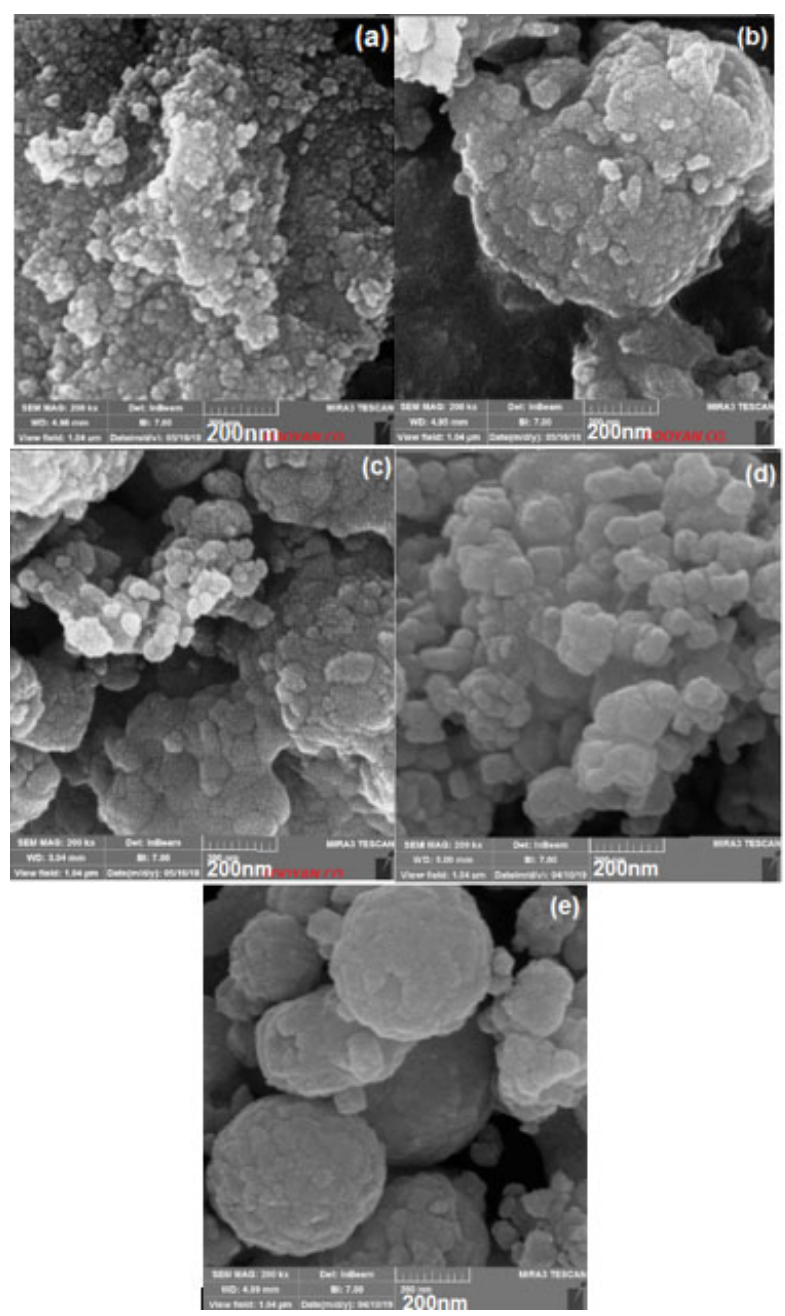

Fig. 8 SEM images of samples prepared by a: Merck, b: the Ball mill method (350 rpm), c: the Ball mill method $(500 \mathrm{rpm})$, d: the sol-gel method $\left(\mathrm{T}=1000^{\circ}\right)$, e: the co-precipitation annealed in ammonia atmosphere $\left(\mathrm{T}=1000^{\circ} \mathrm{C}\right)$

\subsection{Nonlinear optics studies}

By the Z-scan method, the nonlinear optical properties of the titanium nitride with different synthesis methods were performed. The Z-scan technique has the advantages of simplicity and high sensitivity; therefore it is an increasingly popular method to measure optical nonlinearities of materials[47]. Using the Z-scan setup, two important nonlinear optics parameters can be determined, both size and sign[46]. This technique is used to measure both the nonlinear absorption coefficient and the nonlinear refractive index. The Z-scan theory can exhibit either a minimum in back focal(valley) followed by a maximum in 

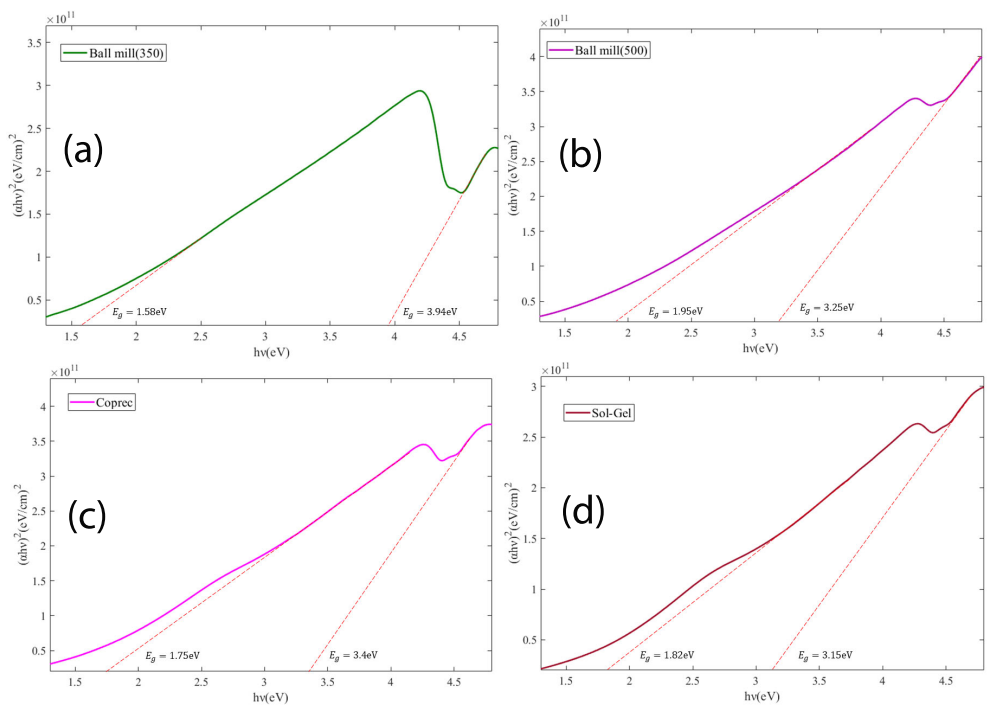

Fig. 9 Variations of $(\alpha h \nu)^{2}$ versus photon energy $(h \nu)$ for samples prepared by a: the Ball mill method(350 rpm), b: the Ball mill method(500 rpm), c: the sol-gel method, d: the co-precipitation.

front focal(peak) or a maximum in back focal (peak) followed by a minimum in the front focal (valley), indicating the positive or negative sign of nonlinear refractive index.

The nonlinear refractive index of materials is defined as follows

$$
n=n_{0}+n_{2} I=n_{0}+\Delta n,
$$

Where $n_{0}$ is the linear refractive index, $I$ is the intensity of beam, and $n_{2}$ is the nonlinear refractive index. To calculate the nonlinear refractive index and nonlinear absorption coefficient, two detectors are used simultaneously, one open aperture and the other closed aperture. The closed aperture detector, nonlinear refractive index, and open aperture detector, nonlinear absorption coefficient of samples are calculated. The nonlinear refractive index is obtained from the following equation[46]

$$
n_{2}=\frac{\delta \Phi_{0}}{k I_{0} L_{e f f}}
$$

where $\Delta \Phi_{0}$ is related to the peak-to-valley of the normalized transmittance obtained through the following equation

$$
\Delta T_{p-v} \simeq 0.406(1-S)^{0.25} \Delta \Phi_{0},
$$

where $S \simeq 0$. Also, $k$ is the wave number, $I_{0}$ is the beam intensity in the focal point, $L_{\text {eff }}=\left(1-\exp \left(-\alpha_{0} L\right)\right) / \alpha_{0}, L$ is the cell thickness, and $\alpha_{0}$ is the linear absorption coefficient. The nonlinear refractive index $n_{2}\left(\mathrm{~cm}^{2} / W\right)$ is obtained from the best fitting performed on the experimental data by using Eq. 3. If 
$n_{2}>0$ self-focusing effect, and if $n_{2}<0$ self-defocussing effect will appear in the sample. In other words, in the closed aperture setup, $n_{2}$ is positive, when the sample movie in the $+\mathrm{z}$ direction from left to right, the transmittance curve, T (z), has a valley-peak. Also, when $n_{2}$ is negative, the light intensity increased, and the transmittance curve, $\mathrm{T}(\mathrm{z})$, has a peak-valley.

Also, the nonlinear absorption coefficient, $\beta$, is obtained by using [47]

$$
\beta=\frac{2^{3 / 2}\left[1-T_{0}\right]}{I_{0} L_{\text {eff }}},
$$

Where $T(0)$ is open-hole transmittance. When $\beta>0$, it is saturable absorption (SA); when $\beta<0$, it is reverse saturable absorption (RSA) or two photon absorption.

In this study, changes in sample synthesis, sample concentration change, light-material interaction are manipulated. By installing different neutral density filters, the samples is illuminated with laser powers of $70 \mathrm{~mW}, 85 \mathrm{~mW}$, $100 \mathrm{~mW}$, and $120 \mathrm{~mW}$. Also, different weight percentages $0.3 \%, 0.6 \%$, and $1.2 \%$ were prepared from the sample. In the first study, by changing the power of the laser beam, using the neutral density filter, and at a constant concentration of the sample, the nonlinear optical behavior was determined for the synthesized nanoparticles in different methods. In the second study, by changing the concentration of the sample with different weight percentages, the nonlinear behavior of the synthesized nanoparticles by different methods was investigated. The nonlinear properties of TiN prepared by the Ball mill (350rpm), Ball mill (500rpm)co-precipitation, and sol-gel methods in different intensities and concentrations are shown in Figures 10, 11, 12, and 13. The results show well the change in the nonlinear behavior of TiN nanoparticles by changing the concentration, laser beam intensity and synthesis of nanoparticles. In each figure, up is the close aperture results and down is the open aperture data results. The valley, followed by a peak normalized transmittance obtain from the closed aperture Z-scan data indicates that the sign of the refraction index nonlinearity is positive (i.e.self-focusing). This behavior is observed in all data. Also, The valley at the focal point obtain from the open aperture Z-scan data in intensity of $85 \mathrm{~mW}, 100 \mathrm{~mW}$, and $120 \mathrm{~mW}$ indicates that the sign of the nonlinear absorption coefficients is positive (i.e. saturable absorption). But the open aperture Z-scan nonlinear optical curves at $70 \mathrm{~mW}$, shows a peak at the focal point, which indicates that the nonlinear absorption coefficient is negative (i.e.reverse saturable absorption). Therefore, as the figures show, with the increase of the input beam power in the open aperture Z-scan diagrams, we have a change from peak to valley, which shows the change from reverse saturation absorption to saturation absorption.

In the process of absorbing saturation with laser beam radiation to TiN nanoparticles, the electrons in the valence band of this material receive energy and are excited, causing it to go to the conduction band. These electrons continue this process until the conduction band becomes saturated. After the conduction band is saturated, the band electrons do not absorb the energy 
Table 2 The values of nonlinear absorption coefficient and nonlinear refractive index of the sample prepared by the ball mill method (350rpm)

\begin{tabular}{|c|ccc|ccc|}
\hline & \multicolumn{3}{|c|}{$n_{2} \times 10^{-8}\left(\mathrm{~m}^{2} / W\right)$} & \multicolumn{3}{c|}{$\beta \times 10^{-3}(\mathrm{~m} / W)$} \\
$P_{\text {laser }}$ & $\mathrm{C}=0.3 \%$ & $\mathrm{C}=0.6 \%$ & $\mathrm{C}=1.2 \%$ & $\mathrm{C}=0.3 \%$ & $\mathrm{C}=0.6 \%$ & $\mathrm{C}=1.2 \%$ \\
\hline $70 \mathrm{~mW}$ & 8.73 & 4.11 & 1.92 & -0.89 & -0.36 & -0.15 \\
\hline $85 \mathrm{~mW}$ & 7.53 & 3.58 & 1.75 & 5.87 & 3.14 & 1.81 \\
\hline $100 \mathrm{~mW}$ & 7.09 & 3.18 & 1.57 & 5.65 & 2.94 & 1.61 \\
\hline $120 \mathrm{~mW}$ & 7.07 & 2.82 & 1.35 & 4.97 & 2.74 & 1.40 \\
\hline
\end{tabular}

Table 3 The values of nonlinear absorption coefficient and nonlinear refractive index of the sample prepared by the ball mill method (500rpm)

\begin{tabular}{|c|ccc|ccc|}
\hline & \multicolumn{3}{|c|}{$n_{2} \times 10^{-8}\left(\mathrm{~m}^{2} / W\right)$} & \multicolumn{3}{c|}{$\beta \times 10^{-3}(\mathrm{~m} / \mathrm{W})$} \\
$P_{\text {laser }}$ & $\mathrm{C}=0.3 \%$ & $\mathrm{C}=0.6 \%$ & $\mathrm{C}=1.2 \%$ & $\mathrm{C}=0.3 \%$ & $\mathrm{C}=0.6 \%$ & $\mathrm{C}=1.2 \%$ \\
\hline $70 \mathrm{~mW}$ & 10.1 & 4.53 & 2.05 & -0.83 & -0.3 & -0.13 \\
\hline $85 \mathrm{~mW}$ & 8.60 & 3.8 & 1.77 & 5.47 & 2.9 & 1.73 \\
\hline $100 \mathrm{~mW}$ & 8.32 & 3.54 & 1.58 & 5.54 & 2.53 & 1.55 \\
\hline $120 \mathrm{~mW}$ & 7.12 & 2.96 & 1.42 & 4.86 & 2.64 & 1.33 \\
\hline
\end{tabular}

capacity, which increases the light passing through the material. The reverse saturation absorption process occurs through the ESA excited state absorption process, in which the electrons of the valence band receive energy and are excited and sent to the conduction band. They go to higher energy states in the conduction band. In the interaction of light with $T i N$, the nonlinear absorption process of this material takes place through a two-photon absorption (TPA) process, in which the electrons of the capacitance band go to a virtual plane by absorbing one photon and by absorbing a second photon. They go to the delivery bar. In the open aperture curve, TiN sample with increasing the power of the incident beam to the material, a state change from peak to valley was observed, which is due to the conversion of saturation absorption behavior to reverse saturation absorption matter. Using Eq. 3 and 5 and determining the peaks and valleys in closed and open aperture diagrams, all the nonlinear refractive index and the nonlinear absorption coefficients of the studied samples were determined separately. The results are given in Tables $2,3,4$, and 5 .

According to Tables 2, 3, 4, and 5 at a concentration of $0.3 \%$, with increasing the intensity of the input beam, the nonlinear refractive index decreases and the nonlinear absorption coefficient decreases. At a concentration of $0.6 \%$, with decreasing the intensity of the input beam, the nonlinear refractive index increases and also the nonlinear absorption coefficient increases. At a concentration of $1.2 \%$, with decreasing the intensity of the input beam, the nonlinear refractive index increases and the nonlinear absorption coefficient also increases. At the lowest intensity $(70 \mathrm{~mW})$, the value of the nonlinear absorption coefficient is negative, which indicates a change to the reverse saturation absorption mode. At $120 \mathrm{~mW}$ intensity, the nonlinear refractive index and nonlinear absorption coefficient decrease with increasing concentration. At an intensity of $100 \mathrm{~mW}$, the nonlinear refractive index, and nonlinear absorp- 
(a)
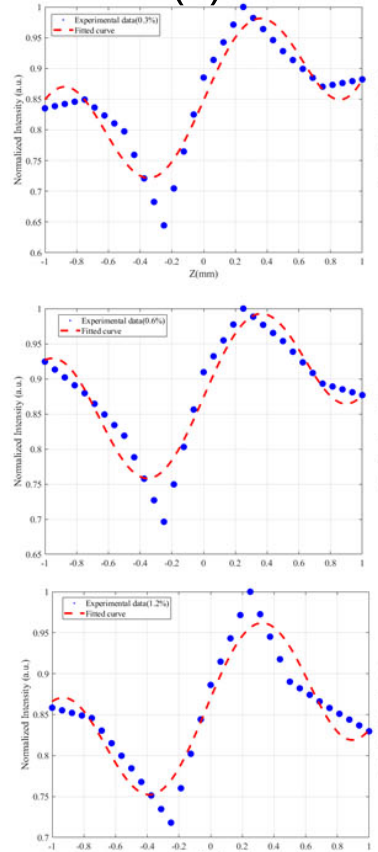

(e)
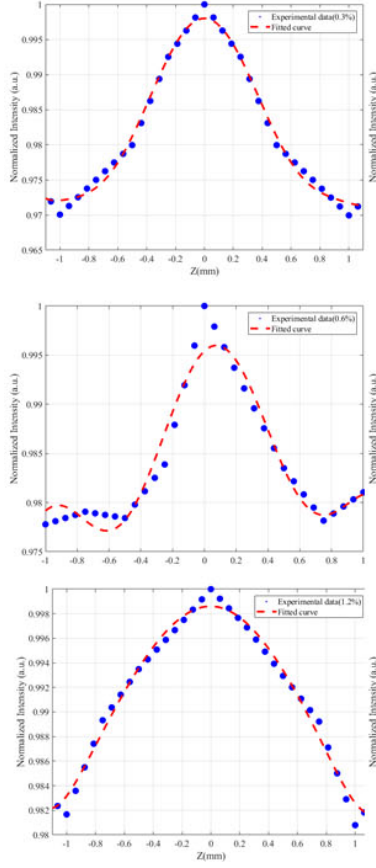

Fig. 10 Nonlinear optical properties of titanium nitride prepared by Ball mill $(350 \mathrm{rpm})$ method. The closed aperture Z-Scan data with different concentration and laser beam power of a: $70 \mathrm{~mW}$, b: $85 \mathrm{~mW}$, c: $100 \mathrm{~mW}$, and $\mathrm{d}: 120 \mathrm{~mW}$, the open aperture Z-Scan data with different concentration and laser beam power of e: $70 \mathrm{~mW}$, f: $85 \mathrm{~mW}$, g: $100 \mathrm{~mW}$, and h: $120 \mathrm{~mW}$.

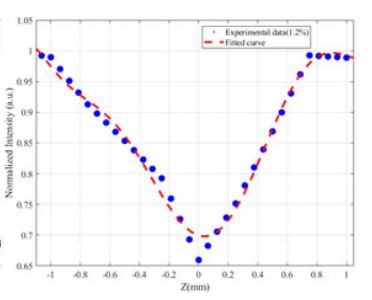

(f)

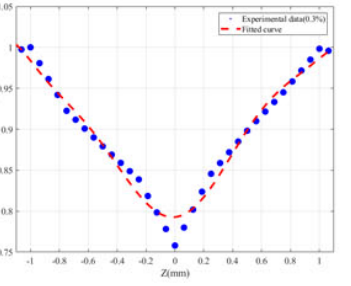

(g)
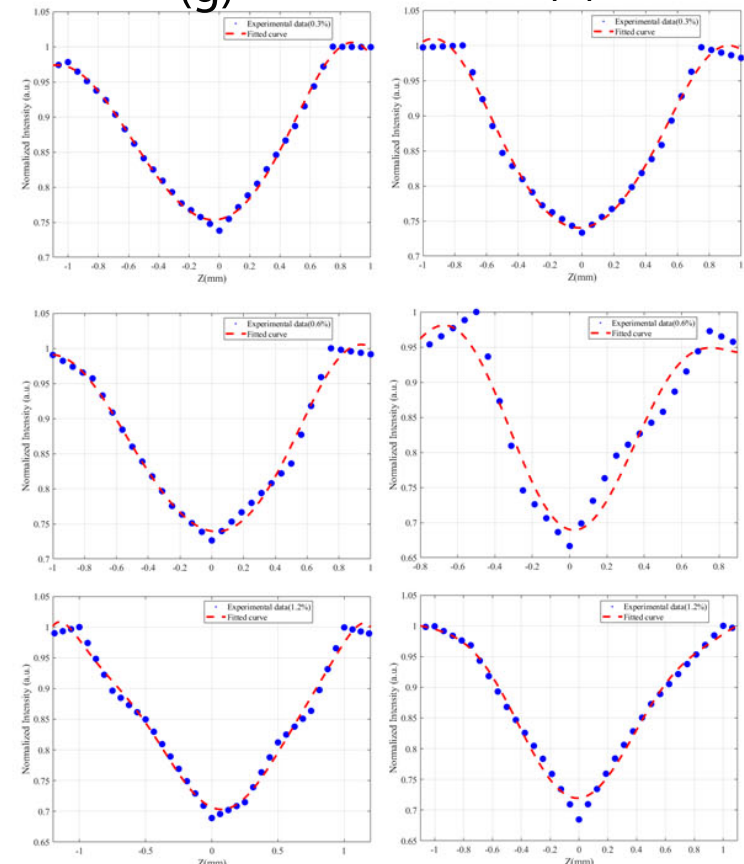

(d)
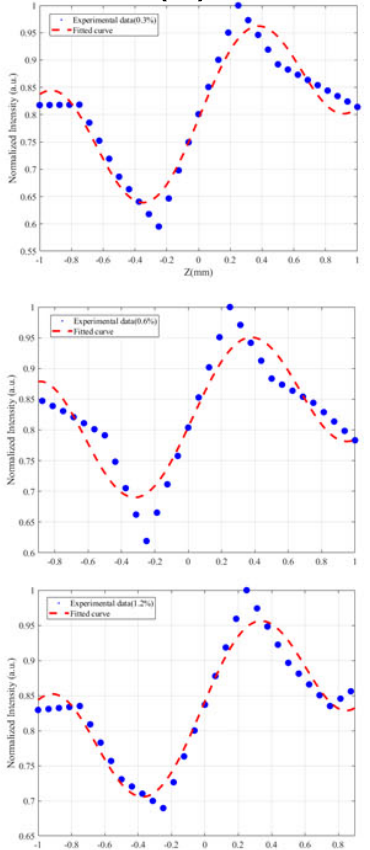

(h)
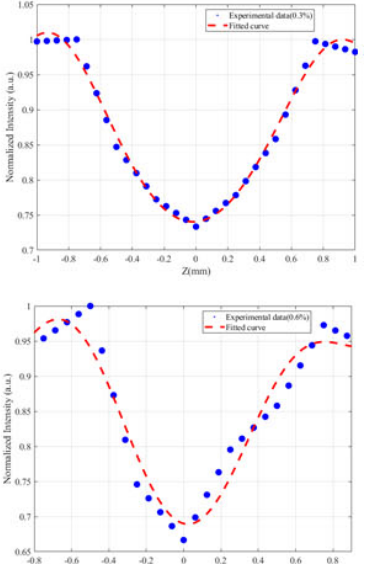
(a)
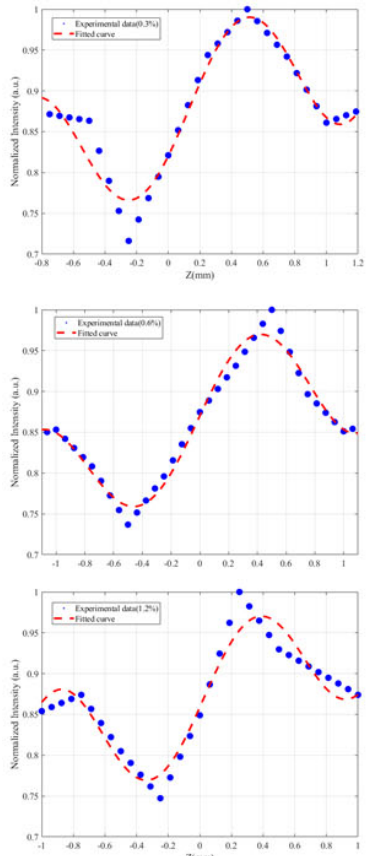

(e)
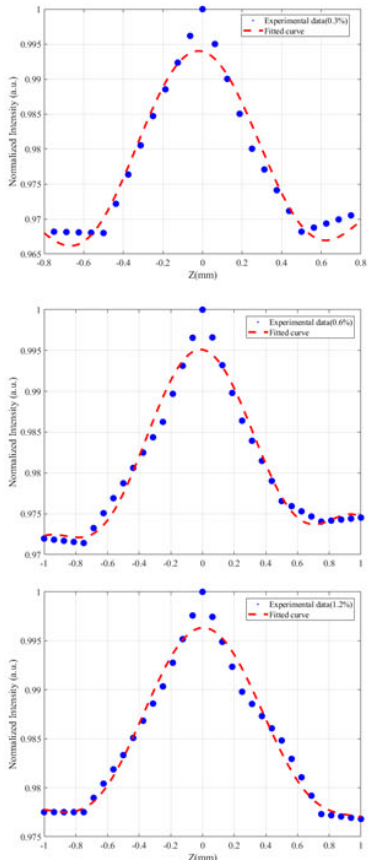

(b)
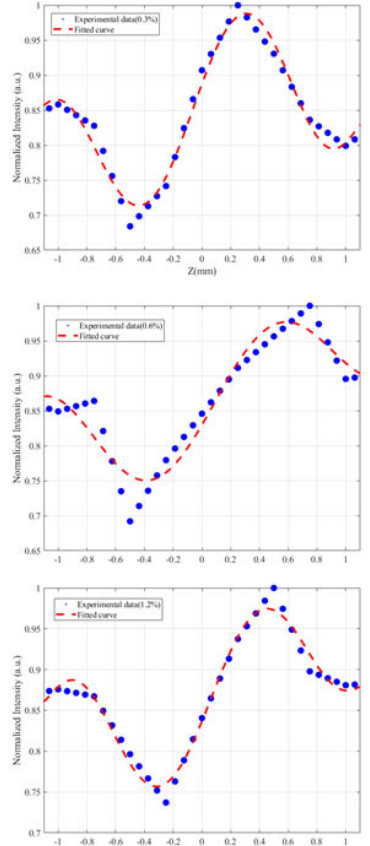

(f)
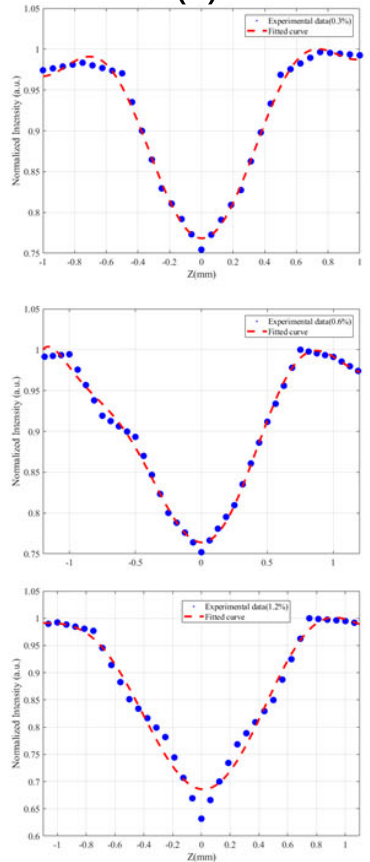

(c)
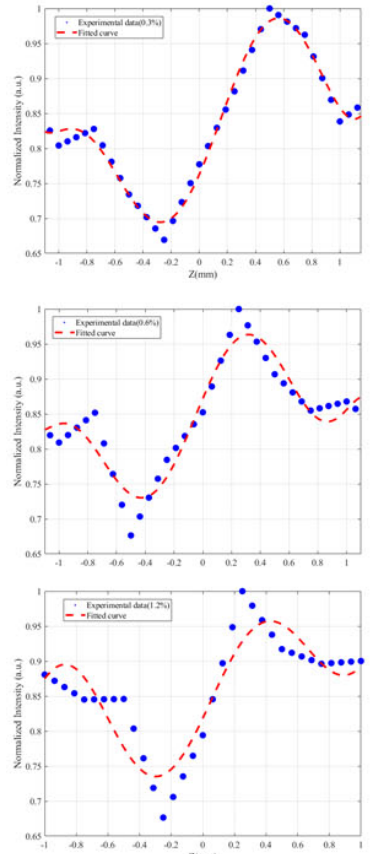

(g)
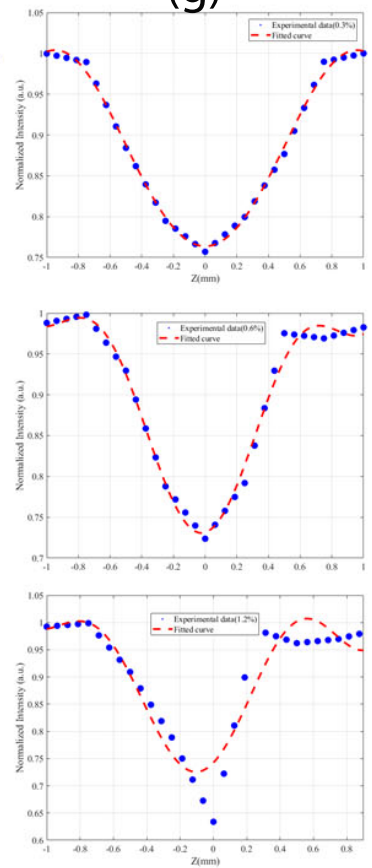

(d)
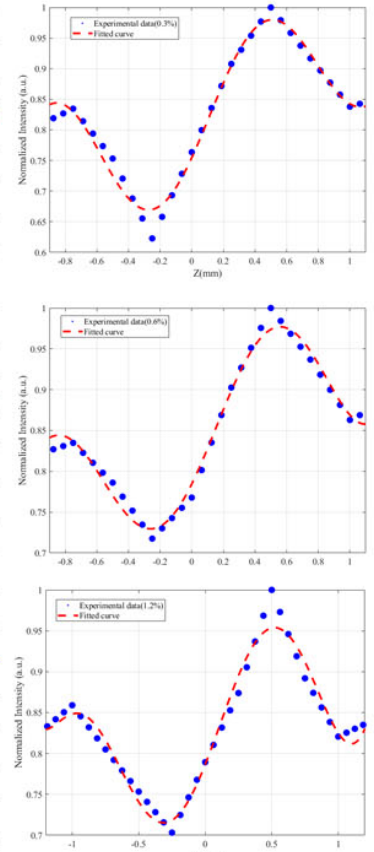

(h)
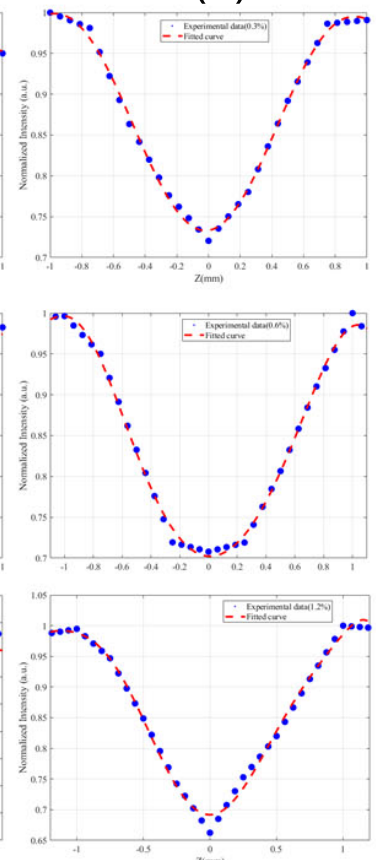

Fig. 11 Nonlinear optical properties of titanium nitride prepared by Ball mill(500 rpm) method. The closed aperture Z-Scan data with different concentration and laser beam power of a: $70 \mathrm{~mW}$, b: $85 \mathrm{~mW}$, c: $100 \mathrm{~mW}$, and d: $120 \mathrm{~mW}$, the open aperture Z-Scan data with different concentration and laser beam power of e: $70 \mathrm{~mW}, \mathrm{f}: 85 \mathrm{~mW}, \mathrm{~g}: 100 \mathrm{~mW}$, and h: $120 \mathrm{~mW}$. 
(a)
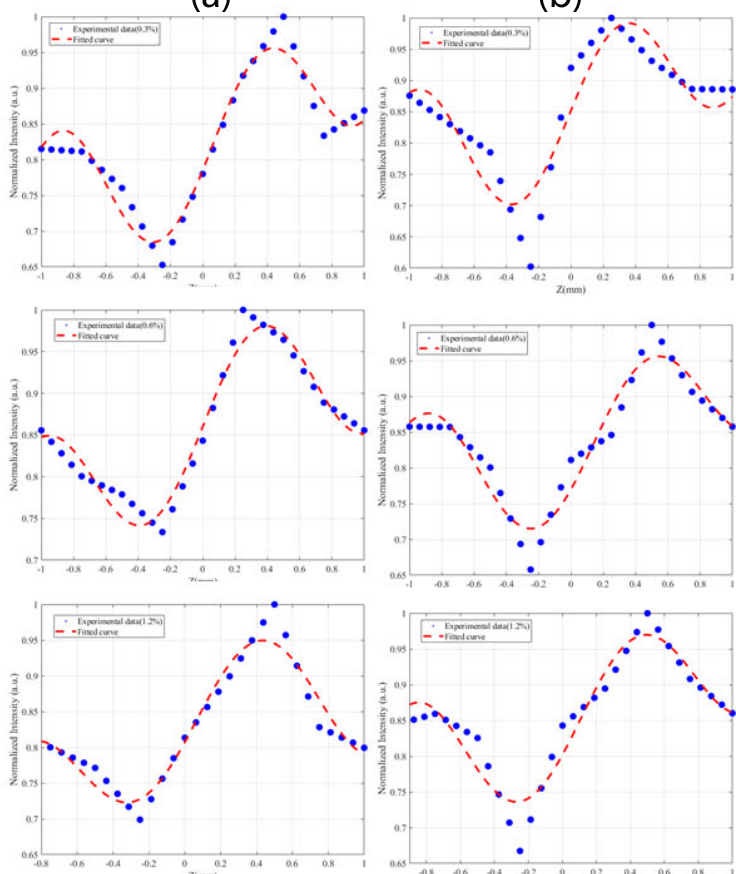

(e)
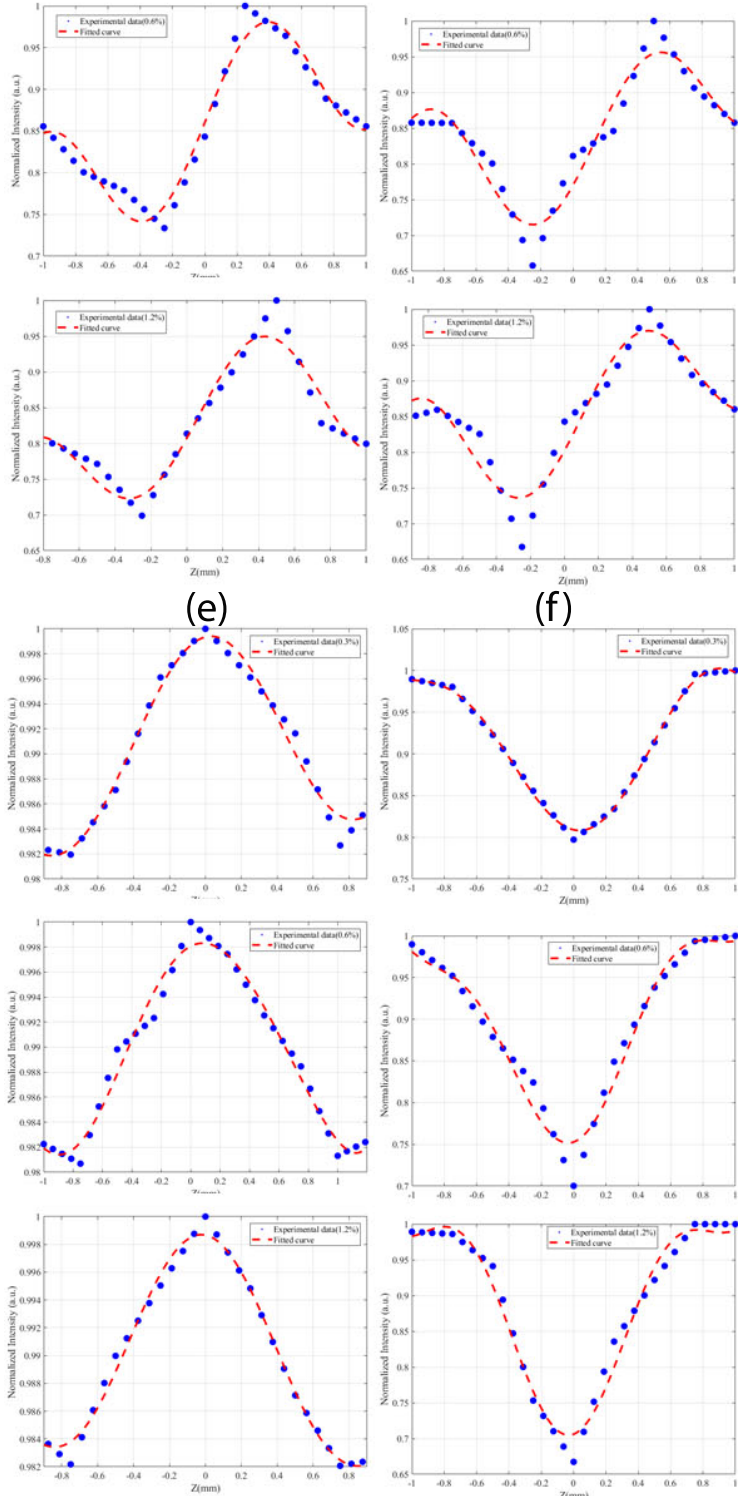

(f)
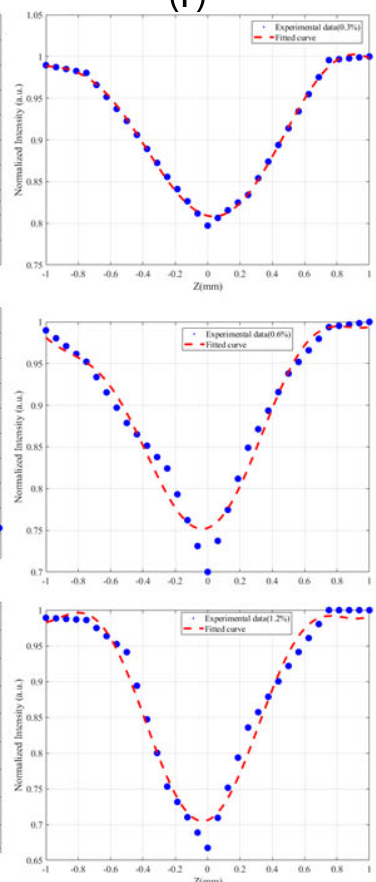

(c)
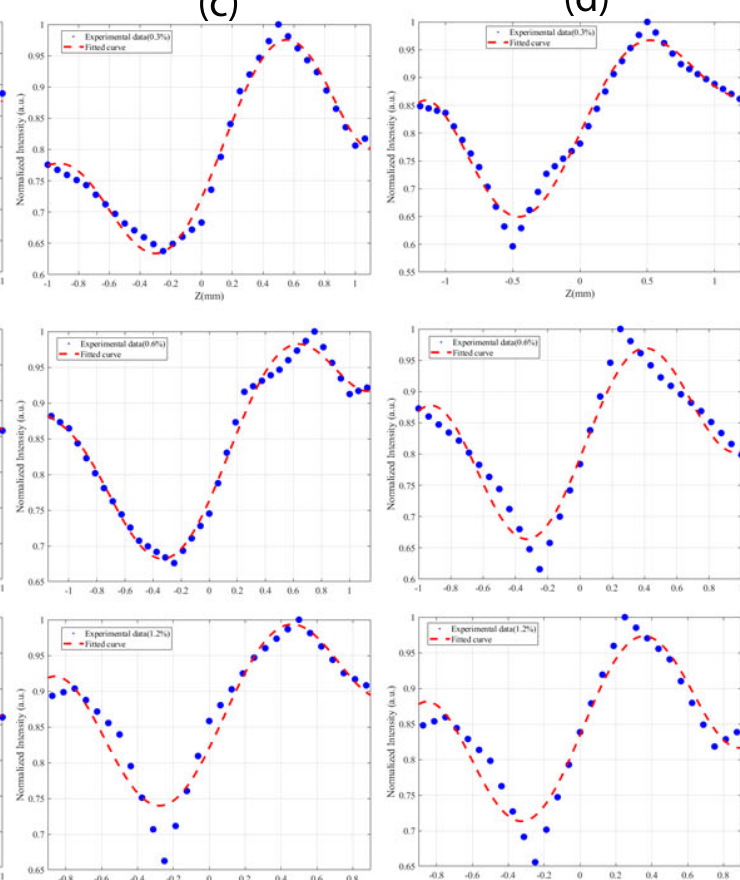

(g)

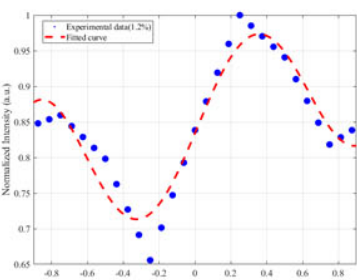

(h)
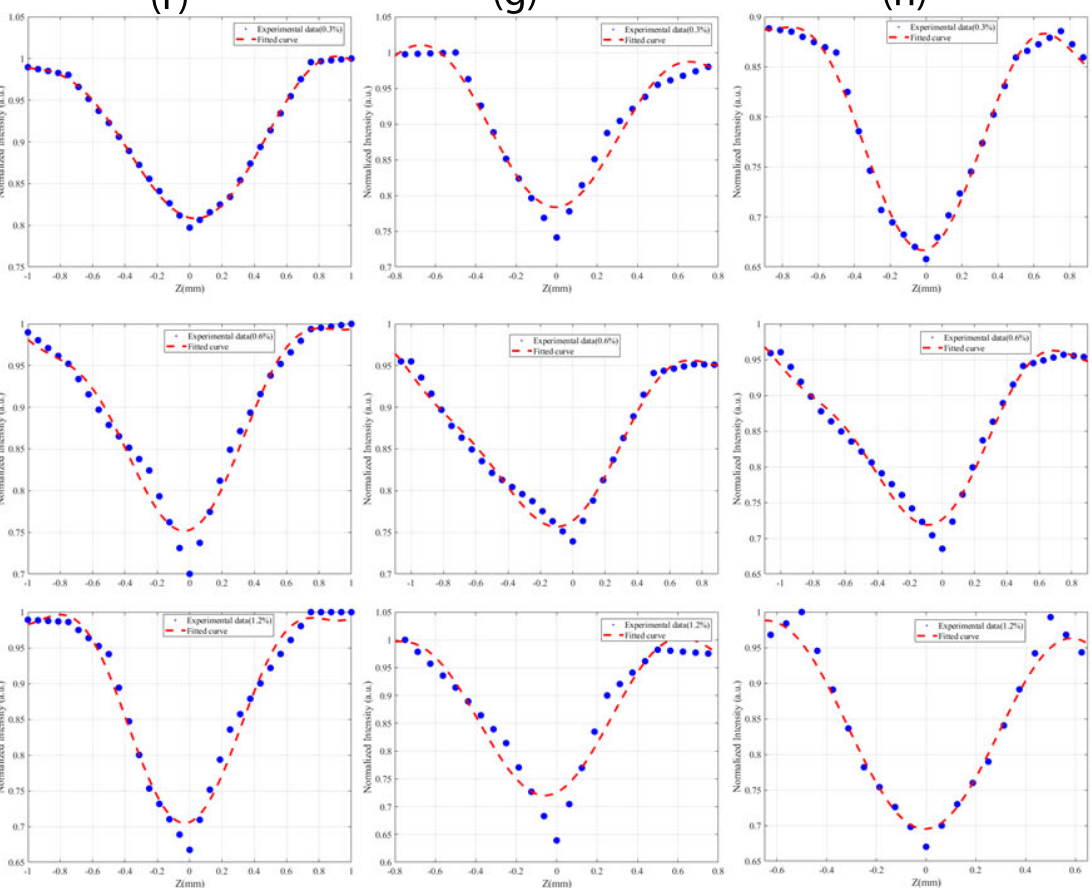

Fig. 12 Nonlinear optical properties of titanium nitride prepared by the co-precipitation method. The closed aperture Z-Scan data with different concentration and laser beam power of a: $70 \mathrm{~mW}$, b: $85 \mathrm{~mW}$, c: $100 \mathrm{~mW}$, and $\mathrm{d}: 120 \mathrm{~mW}$, the open aperture Z-Scan data with different concentration and laser beam power of e: $70 \mathrm{~mW}, \mathrm{f}: 85 \mathrm{~mW}, \mathrm{~g}: 100 \mathrm{~mW}$, and h: $120 \mathrm{~mW}$. 
(a)
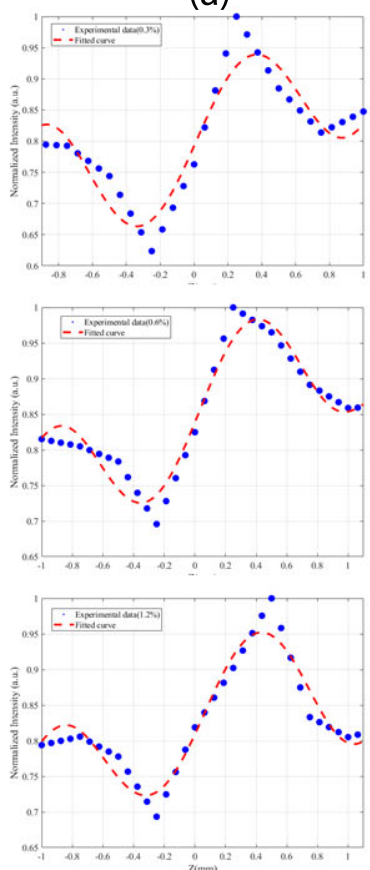

(e)
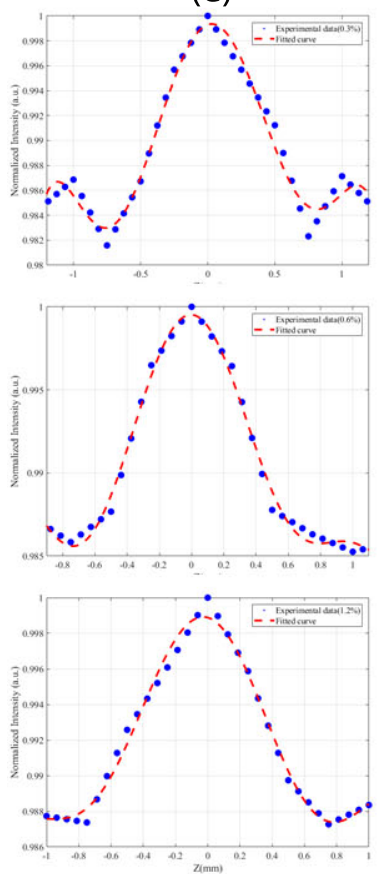

(b)
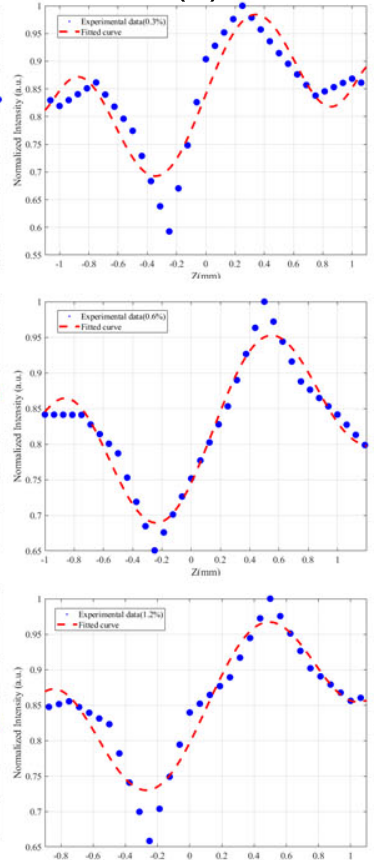

(f)

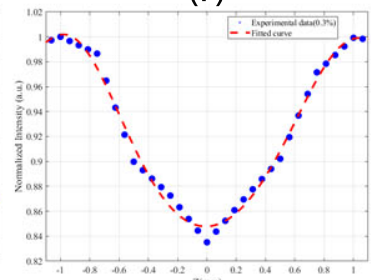

(g)
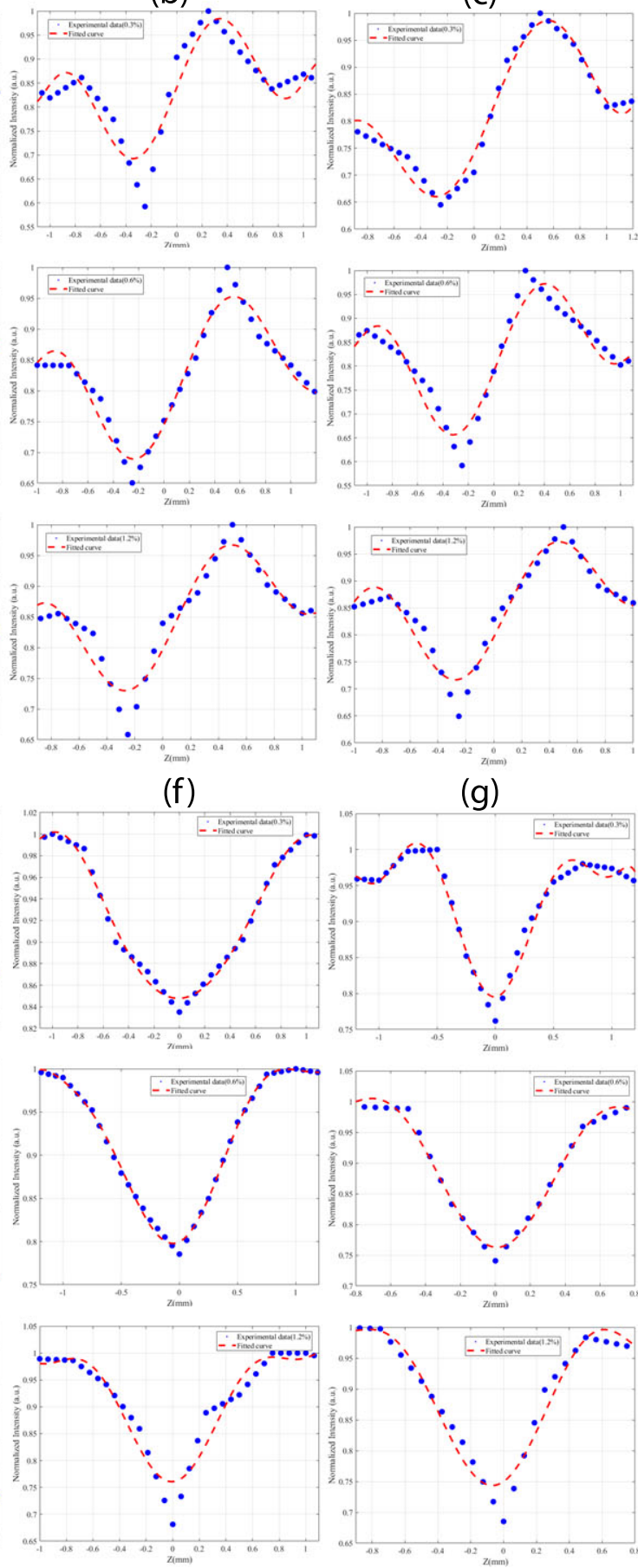

(d)
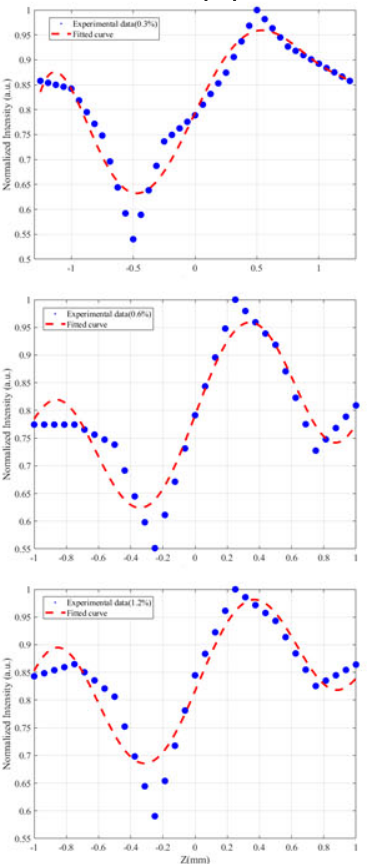

(h)
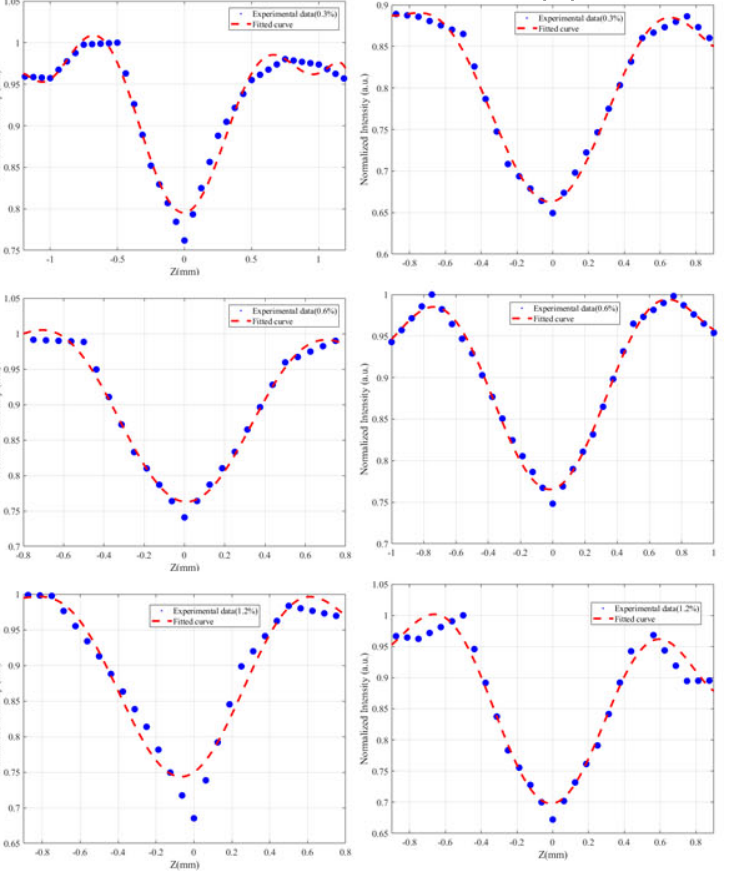

Fig. 13 Nonlinear optical properties of titanium nitride prepared by the sol-gel method. The closed aperture Z-Scan data with different concentration and laser beam power of a: $70 \mathrm{~mW}$, b: $85 \mathrm{~mW}$, c: $100 \mathrm{~mW}$, and d: $120 \mathrm{~mW}$, the open aperture Z-Scan data with different concentration and laser beam power of e: $70 \mathrm{~mW}, \mathrm{f}: 85 \mathrm{~mW}, \mathrm{~g}: 100 \mathrm{~mW}$, and h: $120 \mathrm{~mW}$. 
Table 4 The values of nonlinear absorption coefficient and nonlinear refractive index of the sample prepared by the co-precipitation method.

\begin{tabular}{|c|ccc|ccc|}
\hline & \multicolumn{3}{|c|}{$n_{2} \times 10^{-8}\left(\mathrm{~m}^{2} / W\right)$} & \multicolumn{3}{c|}{$\beta \times 10^{-3}(\mathrm{~m} / \mathrm{W})$} \\
$P_{\text {laser }}$ & $\mathrm{C}=0.3 \%$ & $\mathrm{C}=0.6 \%$ & $\mathrm{C}=1.2 \%$ & $\mathrm{C}=0.3 \%$ & $\mathrm{C}=0.6 \%$ & $\mathrm{C}=1.2 \%$ \\
\hline $70 \mathrm{~mW}$ & 10.6 & 4.67 & 2.21 & -0.56 & -0.26 & -0.11 \\
\hline $85 \mathrm{~mW}$ & 9.31 & 4.43 & 1.88 & 5.12 & 2.79 & 1.62 \\
\hline $100 \mathrm{~mW}$ & 8.59 & 4.17 & 1.68 & 4.56 & 2.62 & 1.42 \\
\hline $120 \mathrm{~mW}$ & 7.30 & 3.48 & 1.47 & 4.15 & 2.29 & 1.25 \\
\hline
\end{tabular}

Table 5 The values of nonlinear absorption coefficient and nonlinear refractive index of the sample prepared by the sol-gel method

\begin{tabular}{|c|ccc|ccc|}
\hline & \multicolumn{3}{|c|}{$n_{2} \times 10^{-8}\left(\mathrm{~m}^{2} / W\right)$} & \multicolumn{3}{c|}{$\beta \times 10^{-3}(\mathrm{~m} / \mathrm{W})$} \\
$P_{\text {laser }}$ & $\mathrm{C}=0.3 \%$ & $\mathrm{C}=0.6 \%$ & $\mathrm{C}=1.2 \%$ & $\mathrm{C}=0.3 \%$ & $\mathrm{C}=0.6 \%$ & $\mathrm{C}=1.2 \%$ \\
\hline $70 \mathrm{~mW}$ & 6.12 & 3.69 & 1.92 & -0.50 & -0.22 & -0.092 \\
\hline $85 \mathrm{~mW}$ & 5.95 & 3.2 & 1.86 & 4.47 & 2.63 & 1.53 \\
\hline $100 \mathrm{~mW}$ & 5.3 & 3.1 & 1.73 & 4.36 & 2.55 & 1.41 \\
\hline $120 \mathrm{~mW}$ & 5.05 & 2.63 & 1.50 & 4.15 & 2.15 & 1.23 \\
\hline
\end{tabular}

tion coefficient decrease with increasing concentration. At $85 \mathrm{~mW}$ intensity, the nonlinear refractive index, and nonlinear absorption coefficient decrease with increasing concentration. At $70 \mathrm{~mW}$, the nonlinear refractive index decreased with increasing concentration, and the nonlinear absorption coefficient increased.

The results show that due to the nonlinear behavior of titanium nitride in $70 \mathrm{~mW}$ is reverse saturation absorption can be to protect the eyes and optical instruments against high intensity laser in low power. Also, due to the nonlinear behavior of titanium nitride at all intensities is self-focusing, can play an important role in the manipulate and correction of the shape of laser pulses. The results in figure 14 show that the Synthesis and concentration functions are a significant help in controlling and manipulating light-matter interactions, provide a very effective way to improve optical properties and thus play an important role in designing and manufacturing new materials for use in optical processes.

\section{Conclusion}

In the present study, the titanium nitride nanoparticles were produced by several methods. The XRD, FTIR, SEM, and UV-Vis techniques were employed to characterize the TiN nanoparticles. The X-ray diffraction pattern revealed the removal of the titanium oxide phase after annealing at $1000^{\circ} \mathrm{C}$ in ammonia. SEM images confirmed the particles aggregation and increase of porousity in the milled samples, due to the decrease in particle size. The Z-scan arrangement was utilized to study the nonlinear optical properties of the samples as a function of laser beam power and concentration of the nanoparticles in dispersed solution. The results show that with increasing concentration and laser beam power, nonlinear refractive index and nonlinear absorption coefficient 

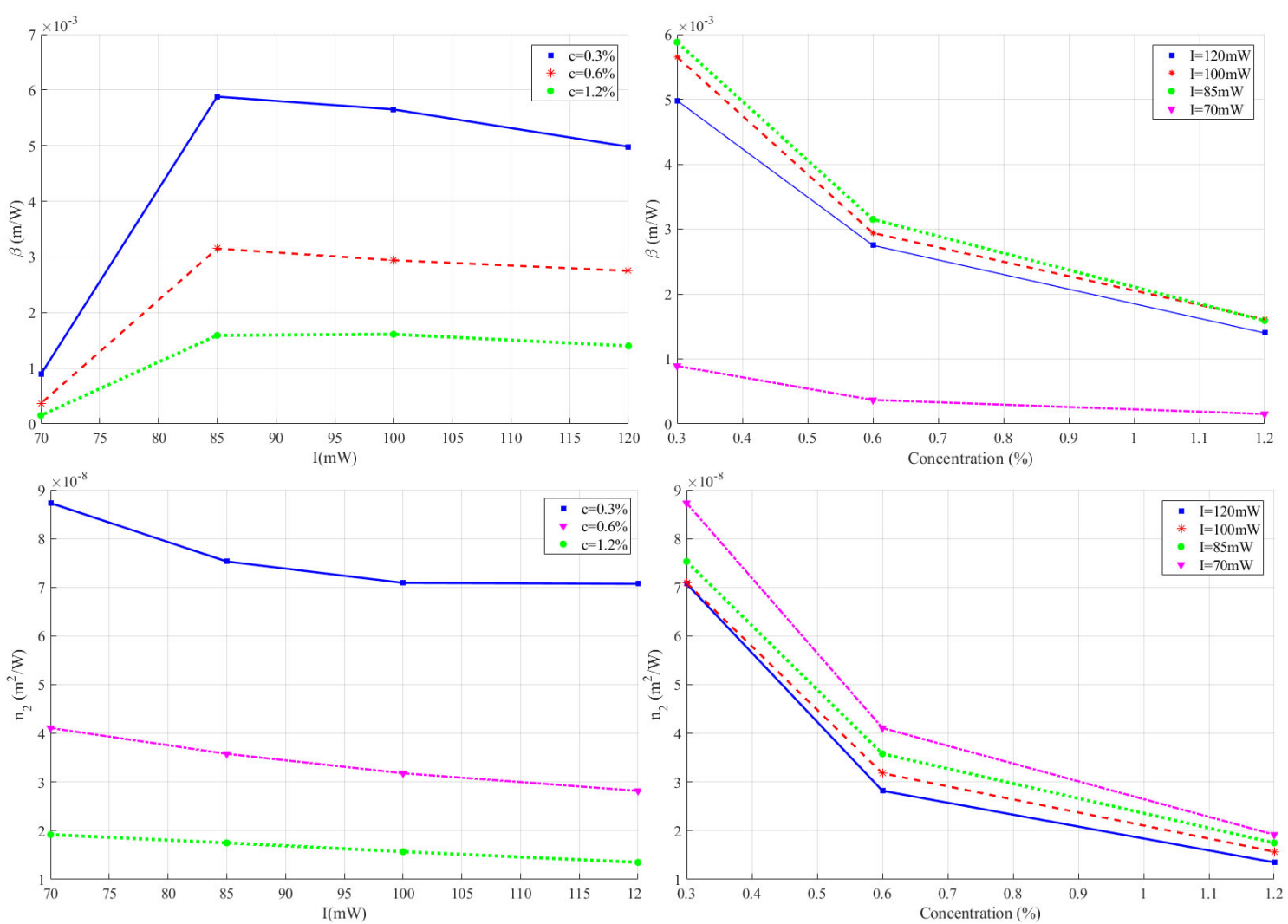

Fig. 14 Plot of nonlinear optical properties $\left(\beta, n_{2}\right)$ against concentration and laser intensity for TiN nanoparticles.

decrease. As the input intensity increased in the nonlinear absorption of the samples, a transition from saturation to reverse saturation was observed. In other words, the value of the nonlinear absorption coefficient at the lowest investigated intensity $(70 \mathrm{~mW})$ results in a negative value which decreases the absorption coefficient. This coefficient was obtained at higher power $(120 \mathrm{~mW}$, $100 \mathrm{~mW}, 85 \mathrm{~mW}$ ), which increased the absorption coefficient. Therefore, the results showed that the nonlinear refractive index behavior of the samples is always similar and has Self-focusing behavior, but the behavior of the nonlinear absorption coefficient at the power of $70 \mathrm{~mW}$ is inverse saturation absorption and at other powers is the saturation absorption.

\section{Conflict of Interest}

The authors declare that they have no conflict of interest. 


\section{References}

1. L. Caspani, and Kaipurath, R. P. M. and Clerici, M. and Ferrera, M. and Roger, T. and Kim, J. and Kinsey, N. and Pietrzyk, M. and Di Falco, A. and Shalaev, V. M. and Boltasseva, A. and Faccio, D., Enhanced Nonlinear Refractive Index in $\epsilon$-Near-Zero Materials, Phys. Rev. Lett., 116, 23, 233901, 5, 2016.

2. S. Luk. Ting, Domenico de Ceglia, Sheng Liu, Gordon A. Keeler, Rohit P. Prasankumar, Maria A. Vincenti, Michael Scalora, Michael B. Sinclair, and Salvatore Campione, Enhanced third harmonic generation from the epsilon-near-zero modes of ultrathin films, Appl. Phys. Lett. 106, 151103, 2015.

3. A. Capretti, Y. Wang, N. Engheta, and L. Dal Negro, Enhanced third-harmonic generation in Si-compatible epsilon-near-zero indium tin oxide nanolayers, Opt. Lett. 40, 1500-1503, 2015.

4. X. Niu, X. Hu, S. Chu, Q. Gong, Epsilon-Near-Zero Photonics: A New Platform for Integrated Devices, Advanced Optical Materials, 6, 1701292, 2018.

5. Minzioni et al, Roadmap on all-optical processing, J. Opt. 21 063001, 2019.

6. K. Sugioka, , Cheng, Y. Ultrafast lasers-reliable tools for advanced materials processing. Light Sci Appl 3, e149, 2014.

7. Ajit P. Joglekar, Hsiao-hua Liu, Edgar Meyhöfer, Gerard Mourou, and Alan J. Hunt, Optics at critical intensity: Applications to nanomorphing, PNAS 101, 16, 5856-5861, 2004.

8. E. N. Glezer, M. Milosavljevic, L. Huang, R. J. Finlay, T. H. Her, J. P. Callan, Threedimensional optical storage inside transparent materials, Optics Letters, 21, 2023, 1999.

9. Luca Rigamonti,Alessandra Forni,Elena Cariati,Gianluca Malavasi andAlessandro Pasini, Solid-State Nonlinear Optical Properties of Mononuclear Copper(II) Complexes with Chiral Tridentate and Tetradentate Schiff Base Ligands, Materials, 12, 21, 3595, 2019.

10. Zhi-Hai Zhang,Jian-Hui Yuan,Kang-Xian Guo, and Elmustapha Feddi, Effect of Conduction Band Non-Parabolicity on the Nonlinear Optical Properties in GaAs/Ga1-xAlxAs Double Semi-V-shaped Quantum Wells , Materials, 12, 1, 78, 2019.

11. Zhi-Hai Zhang, Jian-Hui Yuan, and Kang-Xian Guo, The Combined Influence of Hydrostatic Pressure and Temperature on Nonlinear Optical Properties of GaAs/Ga0.7Al0.3As Morse Quantum Well in the Presence of an Applied Magnetic Field, Materials, 11, 5, 668, 2018.

12. Shivaji R. Dagdale, Gajanan G. Muley, Synthesis and Characterization of a Novel Nonlinear Optical Material $\mathrm{Mg}_{2} \mathrm{Na}_{2} \mathrm{ZnB}_{4} \mathrm{O}_{10}$, Procedia Technology, 24, 682-688, 2016

13. Guo, B., Xiao, Q., Wang, S., Zhang, H., 2D Layered Materials: Synthesis, Nonlinear Optical Properties, and Device Applications. Laser and Photonics Reviews, 13, 1800327, 2019.

14. Michel Sliwa, Sylvie Létard, Isabelle Malfant, Martine Nierlich, Pascal G. Lacroix, Tsuyoshi Asahi, Hiroshi Masuhara, Pei Yu, and Keitaro Nakatani, Design, Synthesis, Structural and Nonlinear Optical Properties of Photochromic Crystals: Toward Reversible Molecular Switches, Chem. Mater., 17, 18, 4727-4735, 2005.

15. GENENE TESSEMA, GIRMA HAILU and MALIK MAAZA, CONCENTRATION DEPENDENT OPTICAL PROPERTIES OF PORPHYRINS IN NAFION MATRIX, Journal of Nonlinear Optical Physics and Materials, 20, 02, 175-182, 2011.

16. Hendry I. Elim, Robinson Anandakathir, Rachel Jakubiak, Long Y. Chiang, Wei Ji, and Loon-Seng Tan, Large concentration-dependent nonlinear optical responses of starburst diphenylaminofluorenocarbonyl methano[60]fullerene pentads, J. Mater. Chem., 17, 1826-1838, 2007.

17. Yalin Huang, Yunle Gu, Mingtao Zheng, Zilin Xu, Wen Zeng, Yingliang Liu, Synthesis of nanocrystalline titanium nitride by reacting titanium dioxide with sodium amide, Materials Letters, 61, 4-5, 1056-1059, 2007.

18. Sajid Ali Ansari, Nazmul Abedin Khan, Zubair Hasan, A. A. Shaikh, Farhana K. Ferdousi, Hasi Rani Barai, Nasrin Siraj Lopae, and Md. Mahbubur Rahman, Electrochemical synthesis of titanium nitride nanoparticles onto titanium foil for electrochemical supercapacitors with ultrafast charge/discharge, Sustainable Energy Fuels, 4, 2480-2490, 2020 . 
19. White, G., Mackenzie, Kenneth, and Johnston, James., Carbothermal Synthesis of Titanium Nitride. Journal of Materials Science. 27. 4287-4293, 1992.

20. M. Eslamloo-Grami, Z. A. Munir, The synthesis of titanium nitride and titanium carbonitride by self-propagating combustion, The Chemistry of Transition Metal Carbides and Nitrides, 978-94-010-7199-4, 1996.

21. Jianhua Ma, Meining Wu, Yihong Du, Suqin Chen, Guoxing Li, Jianbo Hu, Synthesis of nanocrystalline titanium nitride at low temperature and its thermal stability, Journal of Alloys and Compounds, 476, 1-2, 603-605, 2009.

22. M. Nose, M. Zhou, E. Honbo, M. Yokota, S. Saji, Colorimetric properties of ZrN and TiN coatings prepared by DC reactive sputtering, Surf. Coat. Technol. 142 pp.211-217, 2001.

23. P. Clark, B. Dhandapani, S. Oyama, Preparation and hydrodenitrogenation performance of rhenium nitride, Appl. Catal. A 184 pp.175-180, 1999.

24. Y. Dong, Y. Wu, M. Liu, J. Li, Electrocatalysis on shape-controlled titanium nitride nanocrystals for the oxygen reduction reaction, ChemSusChem 6 pp.2016-2021, 2013.

25. P. Tiku, V. Ramamurthy, Titanium Nitride Antireflection Coating for Textured Polysilicon Solar Cells, Optical Materials Technology for Energy Efficiency and Solar Energy Conversion VIII 1149 pp.200-206, 1989.

26. L. Toth, Transition Metal Carbides and Nitrides, Academic press 79 pp.779-820, 1971.

27. A. Mumtaz, W. Class, Color of titanium nitride prepared by reactive dc magnetron sputtering, Vacuum Science and Technology 20 pp.345-348, 1982.

28. S. Kurtz, R. Gordon, Chemical vapor deposition of titanium nitride at low temperatures, Thin Solid Films 140 pp.277-290, 1986.

29. P. Leclair, P. Berera, J. Moodera, Titanium nitride thin films obtained by a modified physical vapor deposition process, Thin Solid Films 376 pp.9-15, 2000.

30. J. Lackner, W. Waldhauser, R. Berghauser, R. Ebner, B. Major, T. Schöberl, Structural, mechanical and tribological investigations of pulsed laser deposited titanium nitride coatings, Thin Solid Films 453 pp.195-202, 2004.

31. I. Kim, P. Kumta, Hydrazide sol-gel synthesis of nanostructured titanium nitride: precursor chemistry and phase evolution, Journal of Materials Chemistry 13 pp.2028-2035, 2003.

32. Y. Huang, Y. Gu, Synthesis of nanocrystalline titanium nitride by reacting titanium dioxide with sodium amide, Materials Letters 61 pp.1056-1059, 2007.

33. G. Zhao, T. Zhang, J. Wang, G. Han, Electrical and optical properties of titanium nitride coatings prepared by atmospheric pressure chemical vapor deposition, Journal of Non Crystalline Solids 354 pp.1272-1275, 2008.

34. J. Li, L. Gao, J. Sun, Q. Zhang, J. Guo, D. Yan, Synthesis of Nanocrystalline Titanium Nitride Powders by Direct Nitridation of Titanium Oxide, J. Am. Ceram. Soc., 84 pp.3045-47, 2001.

35. H. Zhang, F. Li, Q. Jia, Preparation of titanium nitride ultrafine powders by sol-gel and microwave carbothermal reduction nitridation methods, j.ceramint. 04 pp. 1071-1075, 2009.

36. X. Kan, H. Zhu, C. Deng, C. Yu, Low temperature synthesis of nanoscale titanium nitride via molten-salt-mediated magnesiothermic reduction, 14 pp. 315-325, 2017.

37. I. Kim, N. Kumta, Hydrazide sol-gel process: A novel approach, for synthesizing nanostructured titanium nitride, Materials Science and Engineering 98 pp.123-134, 2003.

38. E. Ramasamy, C. Jo, A. Anthonysamy, I. Jeong, J. Kim, J. Lee, Soft-template simple synthesis of ordered mesoporous titanium nitride-carbon nanocomposite for high performance dye-sensitized solar cell counter electrodes, Chemistry of Materials 24 pp.15751582, 2012.

39. S. Oyama, Introduction to the chemistry of transition metal carbides and nitrides", In The chemistry of transition metal carbides and nitrides, Springer, Dordrecht 36 .pp.1-27, 1996.

40. H. Pierson, Handbook of refractory carbides and nitrides: properties, characteristics, processing and applications, William Andrew 405 pp.115-125, 1996.

41. T. Massalski, Binally Alloy Phase Diagrams, American society for metals 11pp.1020$1125,1986$. 
42. Lili Gui, Shahin Bagheri, Nikolai Strohfeldt, Mario Hentschel, Christine M. Zgrabik, Bernd Metzger, Heiko Linnenbank, Evelyn L. Hu, and Harald Giessen, Nonlinear Refractory Plasmonics with Titanium Nitride Nanoantennas, Nano Lett., 16, 9, 5708-5713, 2016

43. W. Li, U. Guler, N. Kinsey, G. V. Naik, A. Boltasseva, J. Guan, and V. M. Shalaev, Refractory Plasmonics with Titanium Nitride: Broadband Metamaterial Absorber, Adv. Mater. 26(47), 7959-7965, 2014.

44. S. Divya, V. Nampoori, P. Radhakrishnan, and A. Mujeeb, Evaluation of nonlinear optical parameters of TiN/PVA nanocomposite - A comparison between semi-empirical relation and Z-scan results, Curr. Appl. Phys. 14 93-95, 2014.

45. S. Divya, V. P. N. Nampoori, P. Radhakrishnan, and A. Mujeeb, Origin of optical non-linear response in TiN owing to excitation dynamics of surface plasmon resonance electronic oscillations, Laser Phys. Lett. 11(8), 1-7, 2014

46. M. Sheik-bahae; A. A. Said; E. W. Van Stryland, High-sensitivity, single-beam $n_{2}$ measurements. Opt. Lett. 14, 17, 955-957, 1989.

47. A. N. Gheymasi, Y. Rajabi, E. N. Zare, Nonlinear optical properties of poly(aniline-copyrolle)@Zno-based nanofluid, Optical Materials, 102, 109835, 2020.

48. Darabi, H.; Adelifard, M.; Rajabi, Y. Characterization of nonlinear optical refractive index for graphene oxide-Silicon oxide nanohybrid composite, Journal of Nonlinear Optical Physics and Materials, 2019, 28, 01, 1950005.

49. Talebi, S.; Rajabi, Y.; Ardyanian, M. Enhanced nonlinear optical properties of ZnO:WO3 nanocomposites, J. Nanophotonics, 2019, 13, 1, 016003.

50. H. Xinmei; Q. Penglong; Y. Tao; C. Kuo-Chih, Synthesis of titanium nitride nanopowder at low temperature from the combustion synthesized precursor and the thermal stability, journal of Alloys and Compounds,47(4), PP. 838-842(2014).

51. J.I. Pankove, Optical Processes in Semiconductors, PrenticeHall, Englewood Cliffs, (1971).

52. M. N. Solovan, V. V. Brus, E. V. Maistruk, P. D. Maryanchuk, Electrical and optical properties of TiN thin films, Inorganic materials., 50(12),PP. 40-45, 2014.

53. Zheng Xie, Xiangxuan Liu, Peng Zhan, Weipeng Wang, and Zhengjun Zhang, Tuning the optical bandgap of TiO2-TiN composite films as photocatalyst in the visible light, Aip Advances 3, 062129, 2013. 


\section{Figures}
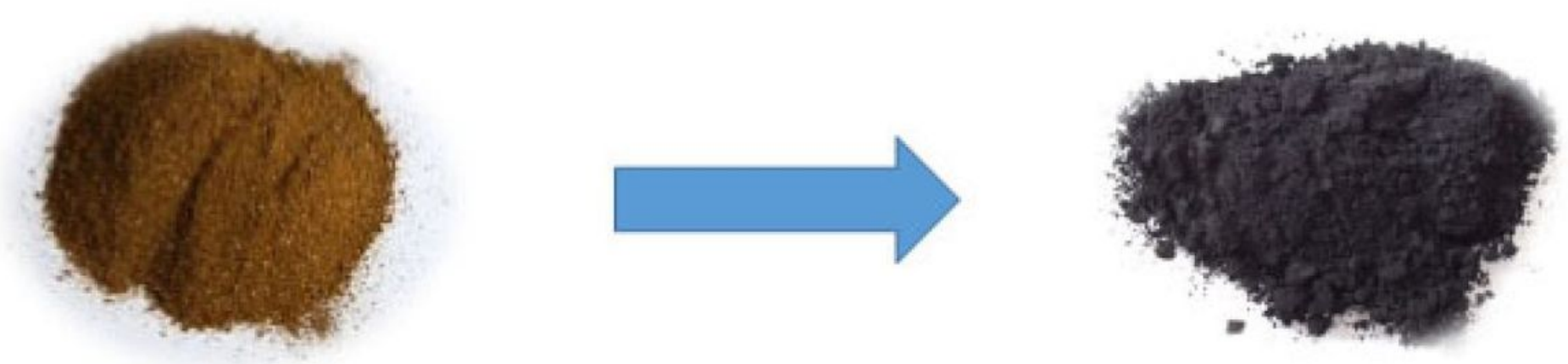

Figure 1

Left: Sample before Ball mill process, Right: resulted sample after Ball mill process.
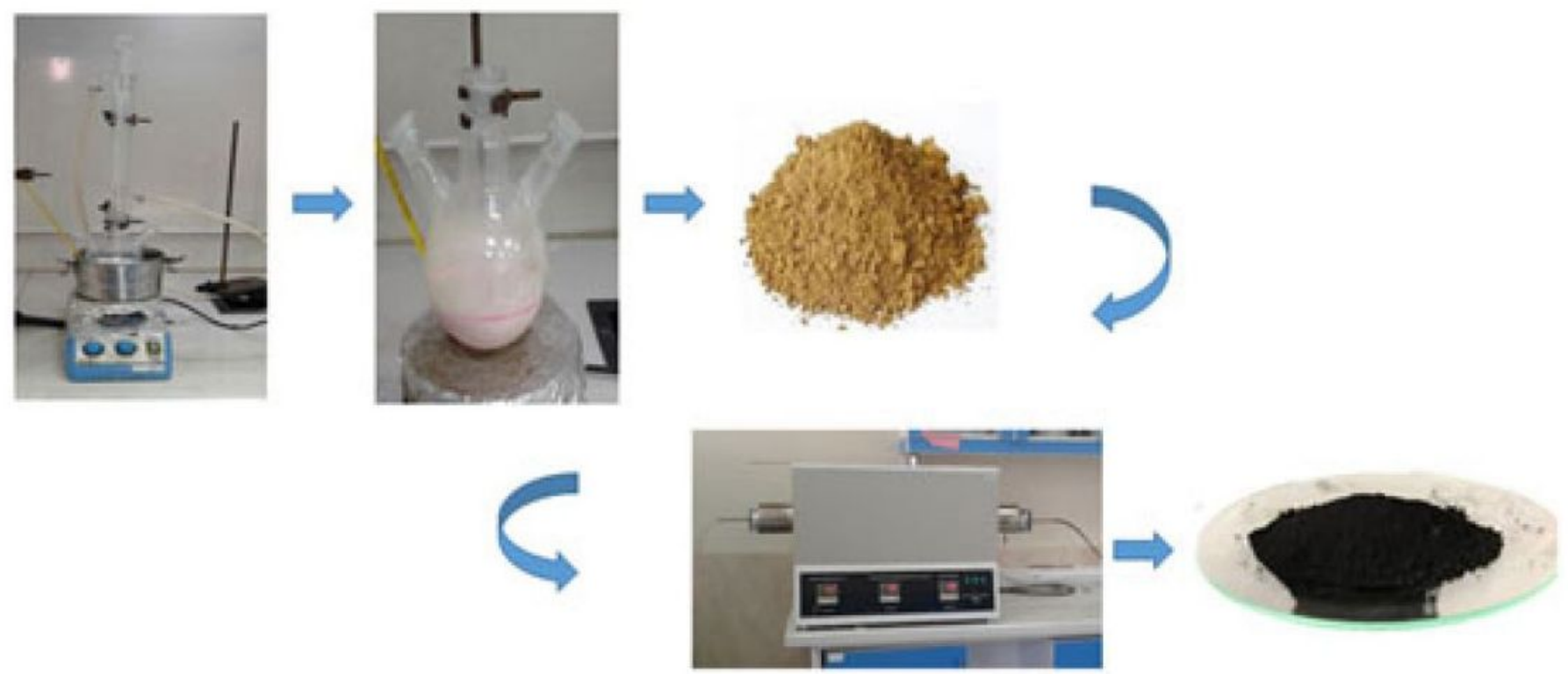

Figure 2

The preparation process of TiN by co-precipitation method. 

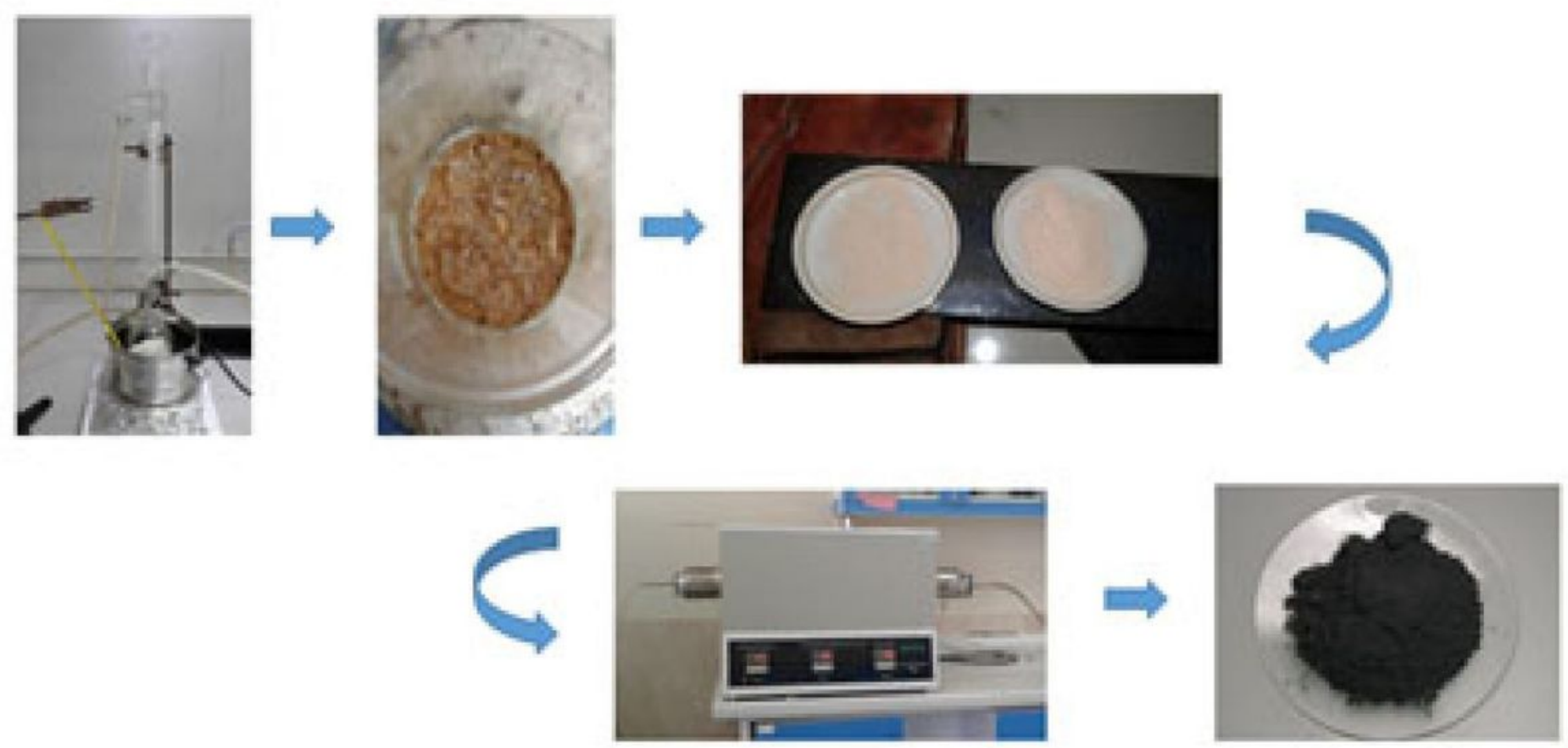

\section{Figure 3}

The preparation process of TiN by sol-gel method.

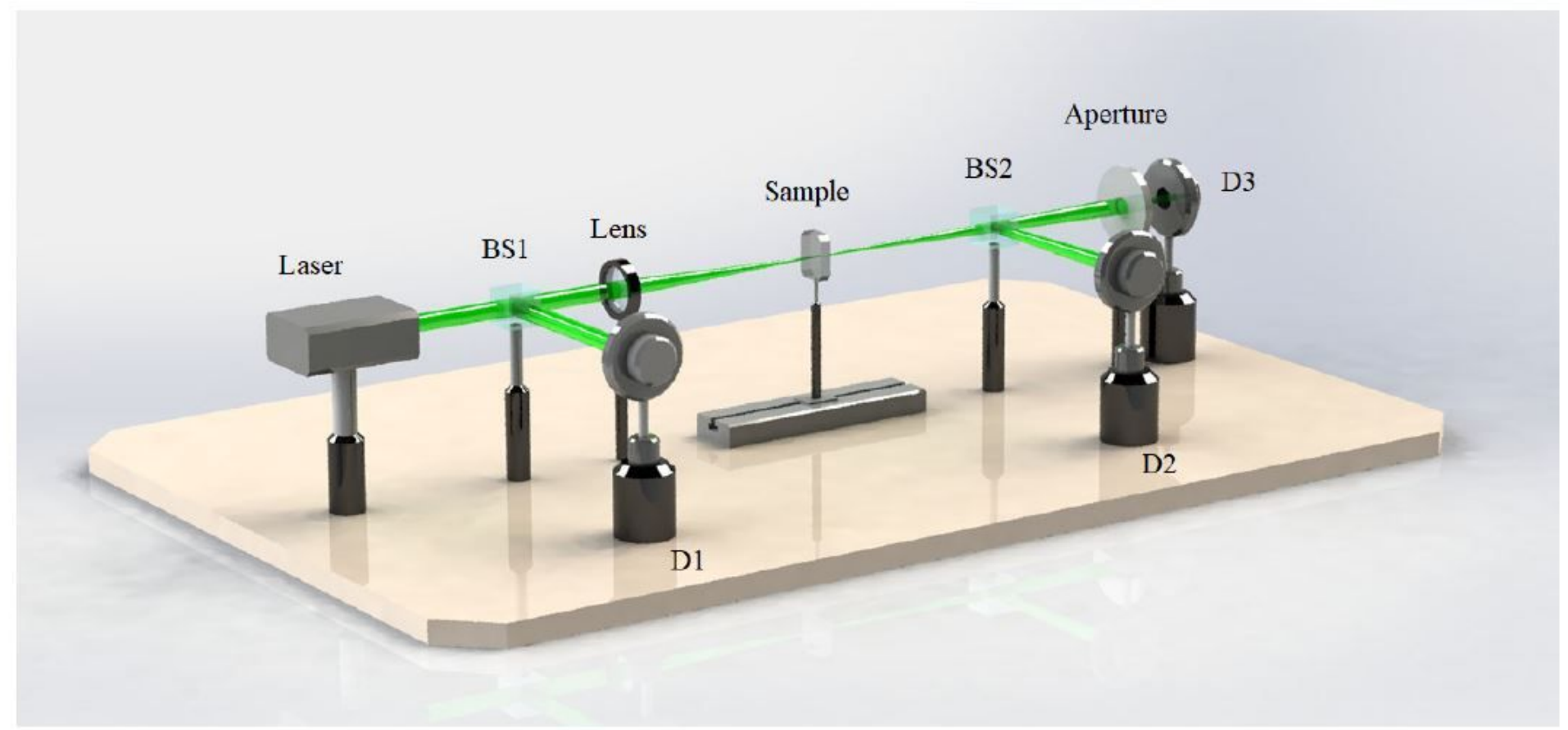

Figure 4

The experimental setup of open-aperture and close-aperture the Z-scan technique: Laser is Nd-Yag, D1, D2 and D3 are intensity detectors, BS1 and BS2 are beam splitter. 

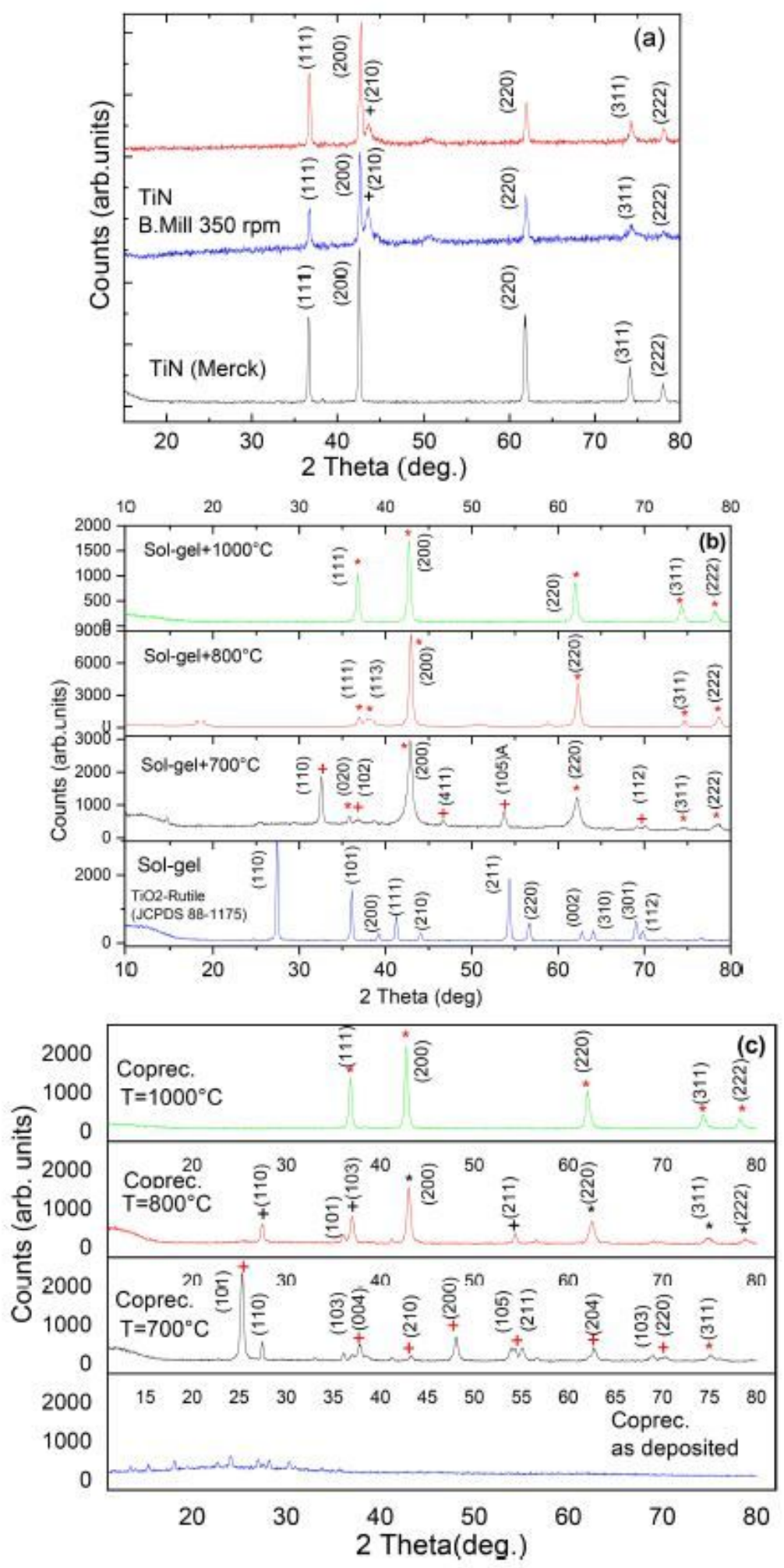

\section{Figure 5}

a - Comparison of the XRD pattern of the sample purchased from Merck, and TiN nanoparticles prepared by Ball. Mill technique. $b$ - The effect of calcination temperature in ammonia atmosphere on the structure of the samples synthesized by the sol-gel method, c - XRD pattern of the sample synthesized by co-precipitation calcinated at different temperatures in ammonia atmosphere, 


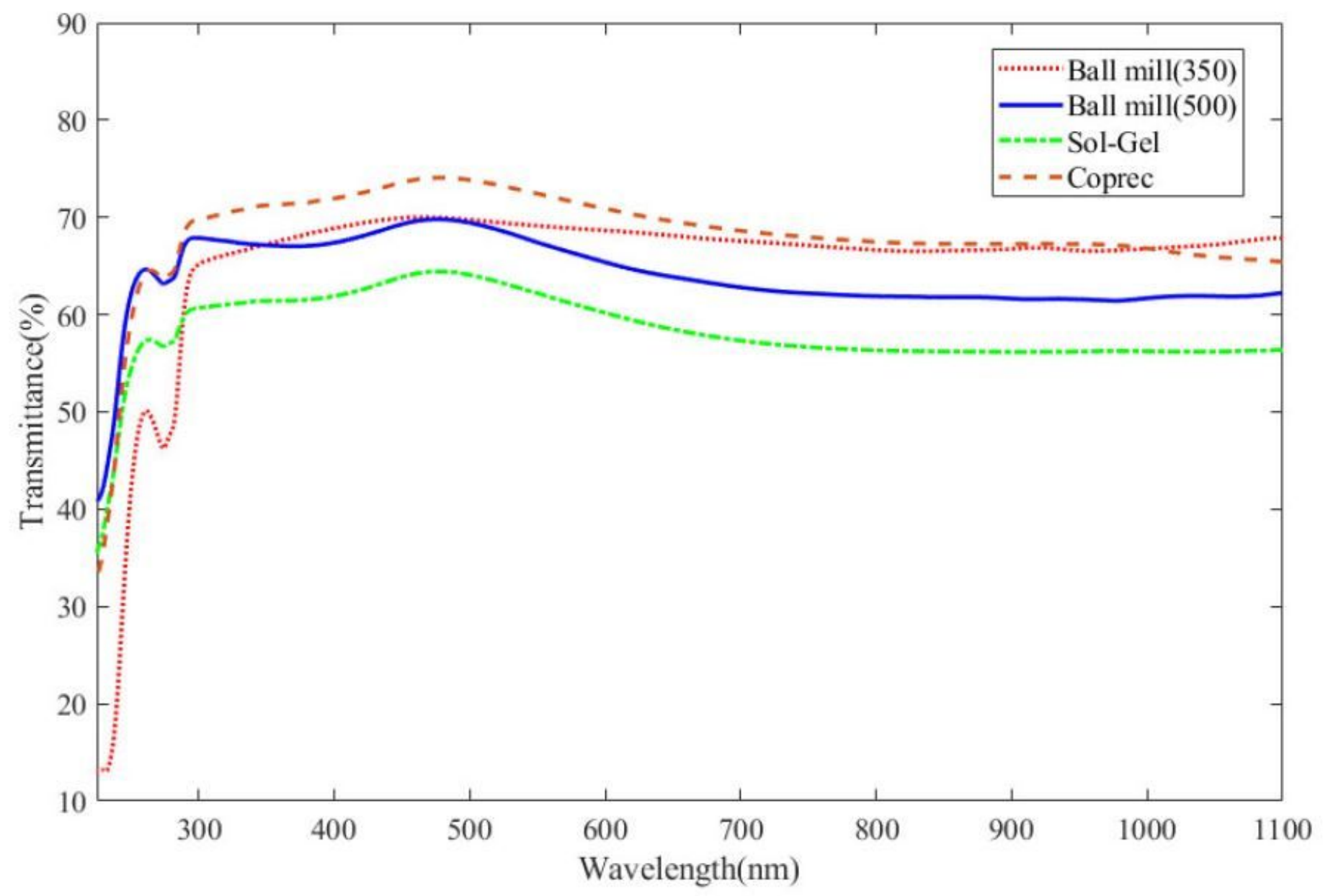

Figure 6

UV-Vis spectra of TiN.

(a)

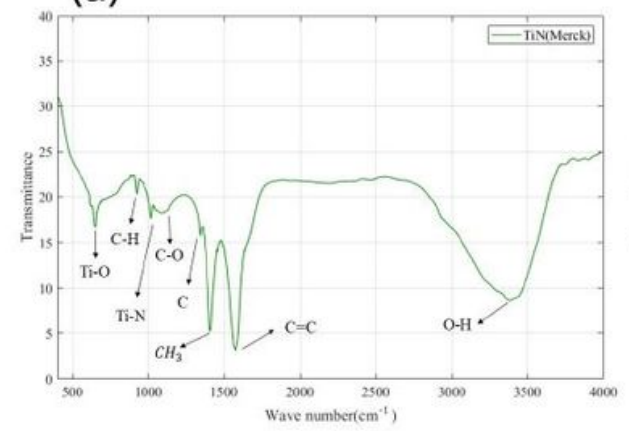

(b)

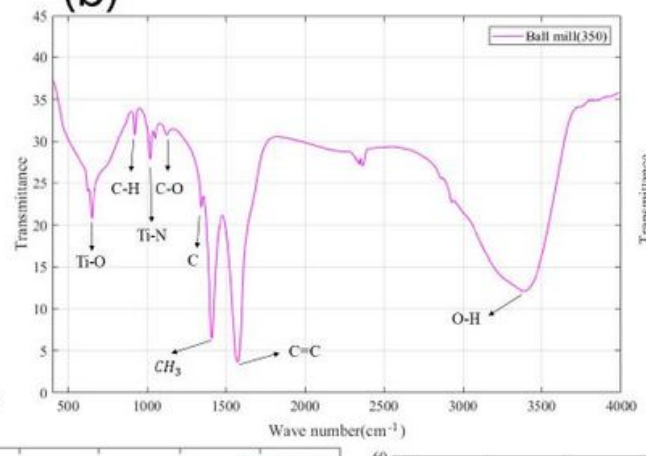

(d)
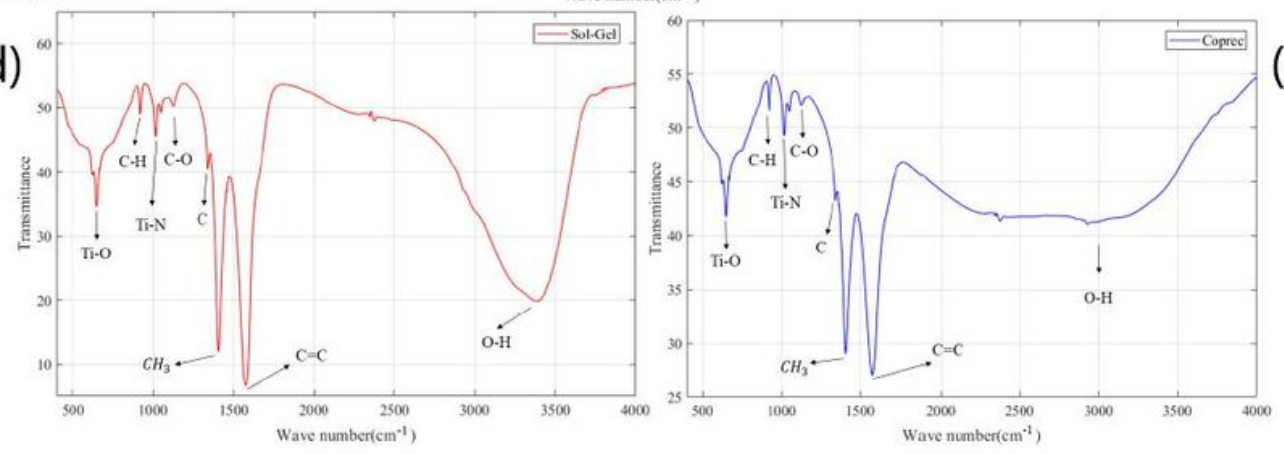

(c)

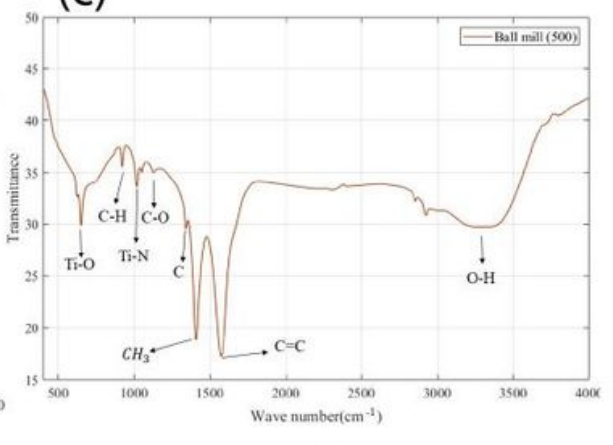

(e) 
Figure 7

FTIR spectra of sample prepared by a: Merck, b: the Ball mill method with 350rpm, c: the Ball mill method with 500rpm, d: the sol-gel method, e: the co-precipitation method.

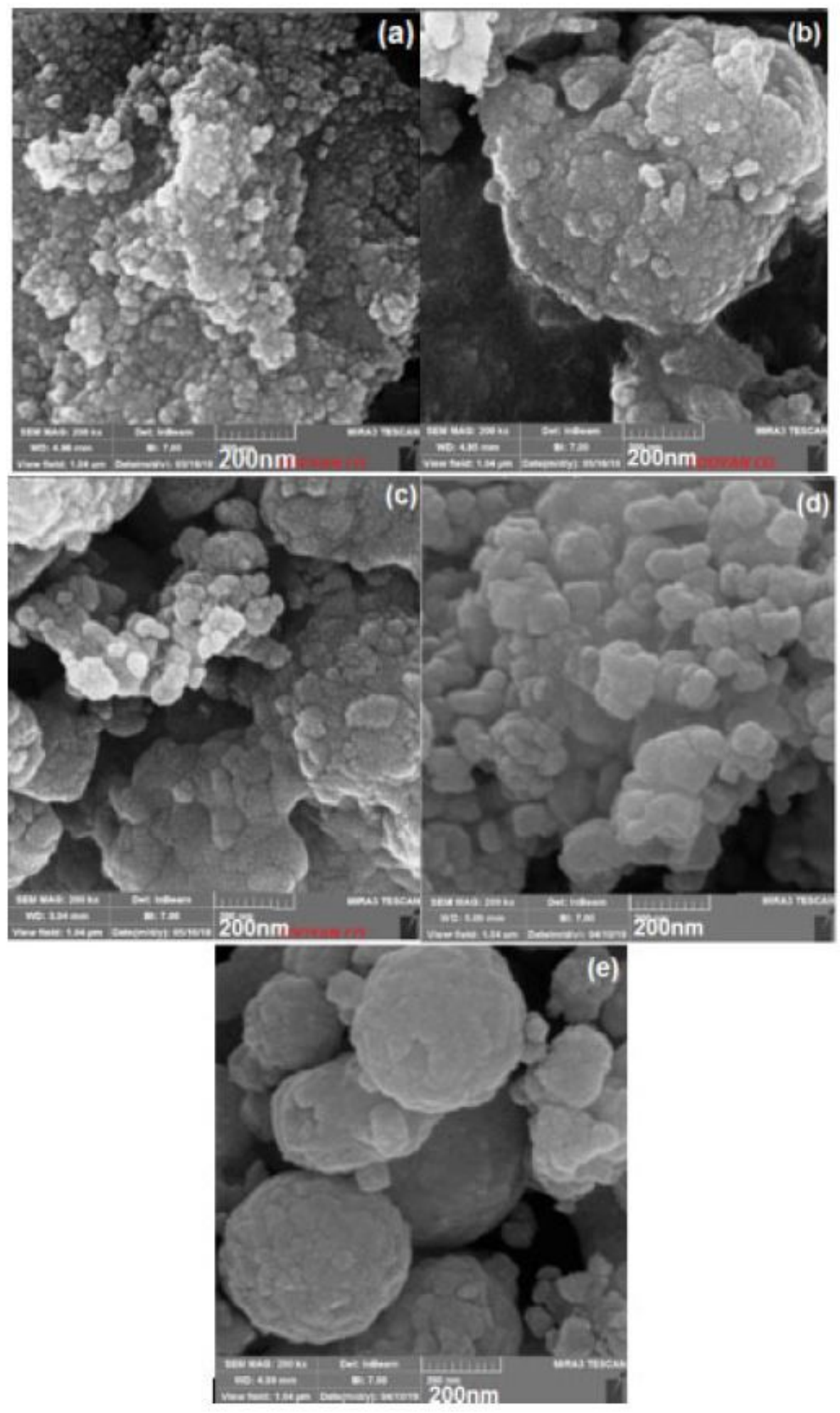

Figure 8 
SEM images of samples prepared by a: Merck, b: the Ball mill method (350 rpm), c: the Ball mill method (500 rpm), d: the sol-gel method $\left(T=1000^{\circ}\right)$, e: the co-precipitation annealed in ammonia atmosphere $\left(\mathrm{T}=1000^{\circ} \mathrm{C}\right)$
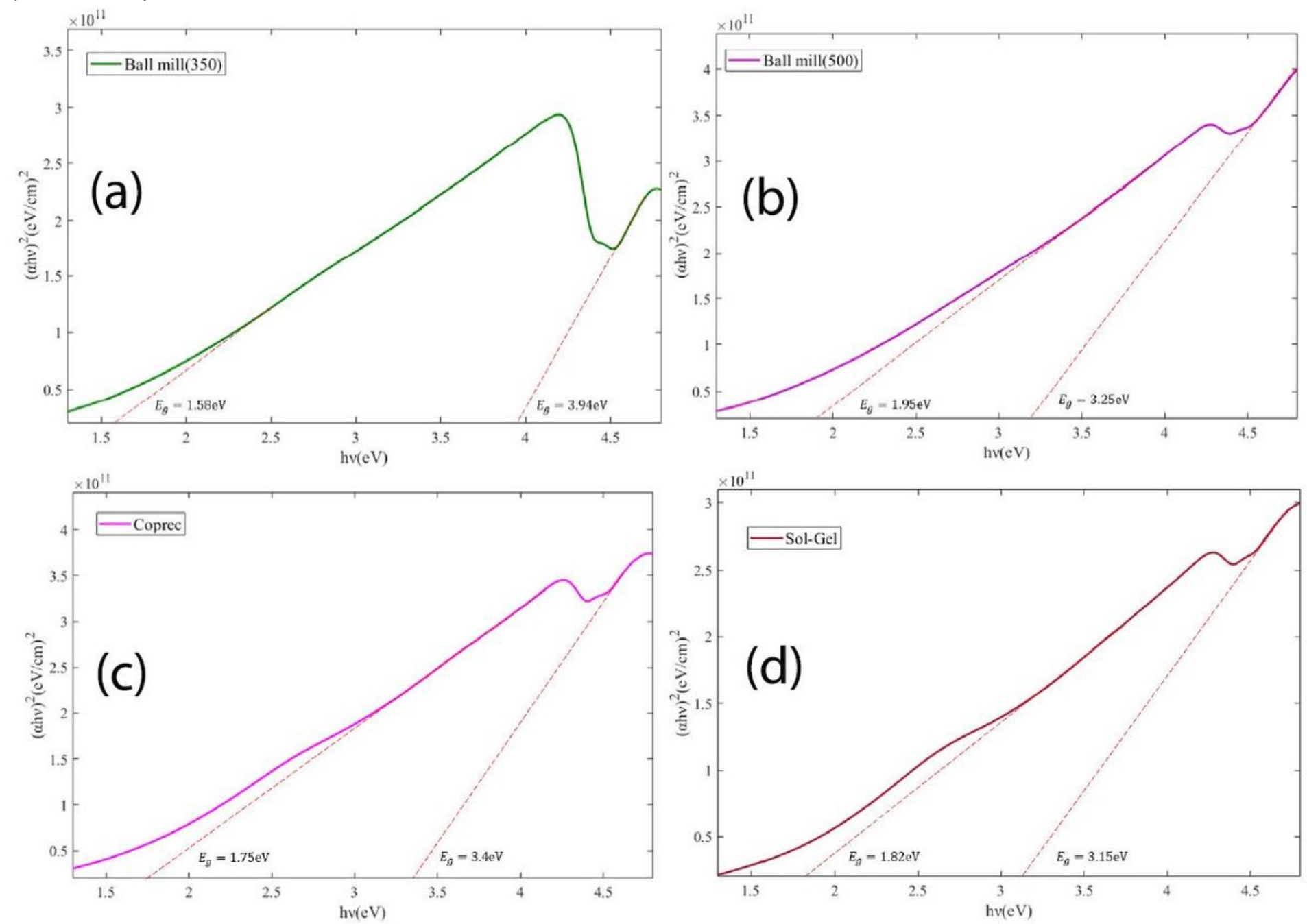

Figure 9

Variations of (ahv)2 versus photon energy (hv) for samples prepared by a: the Ball mill method(350 rpm), b: the Ball mill method(500 rpm), c: the sol-gel method, d: the co-precipitation. 
(a)

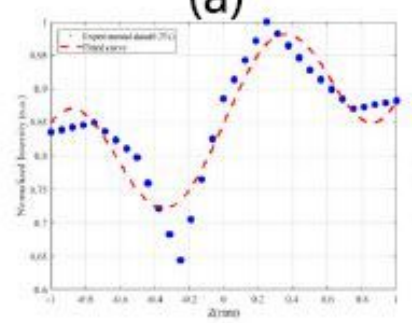

(b)

(c)
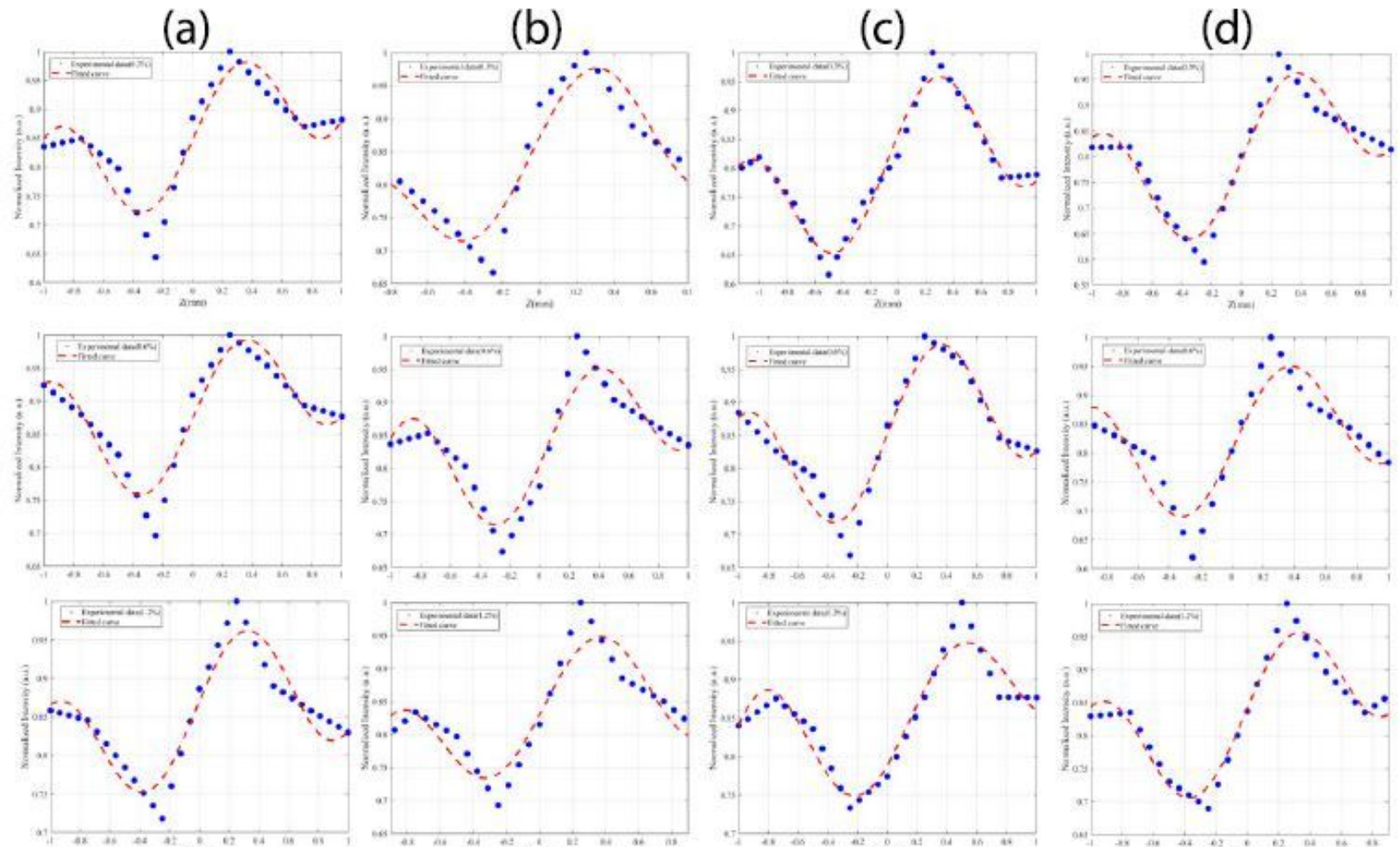

(e)

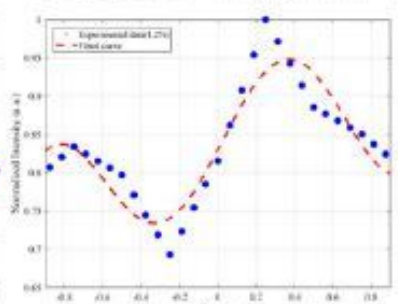

(f)
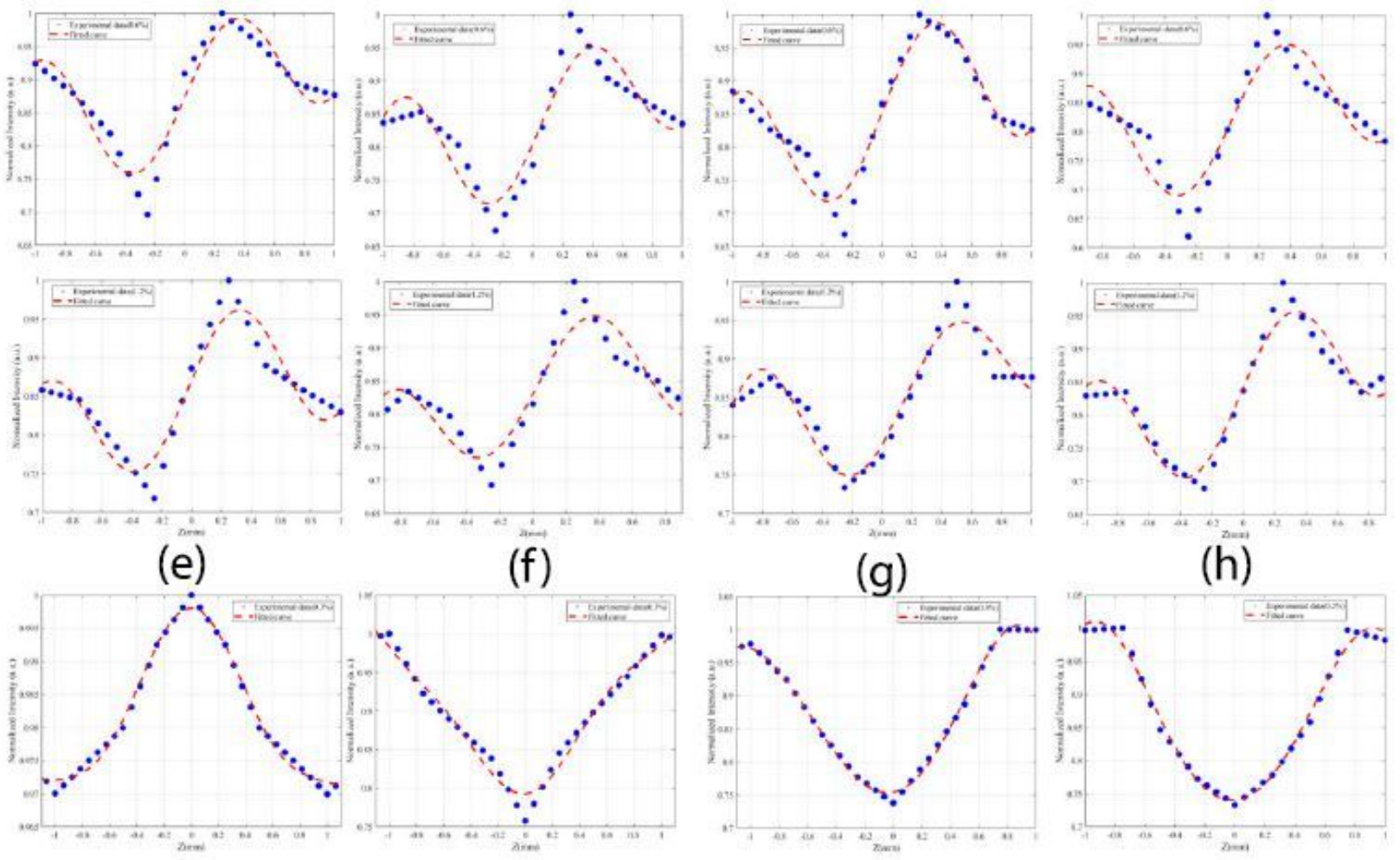

(g)

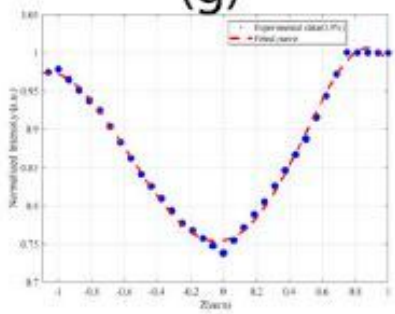

(h)
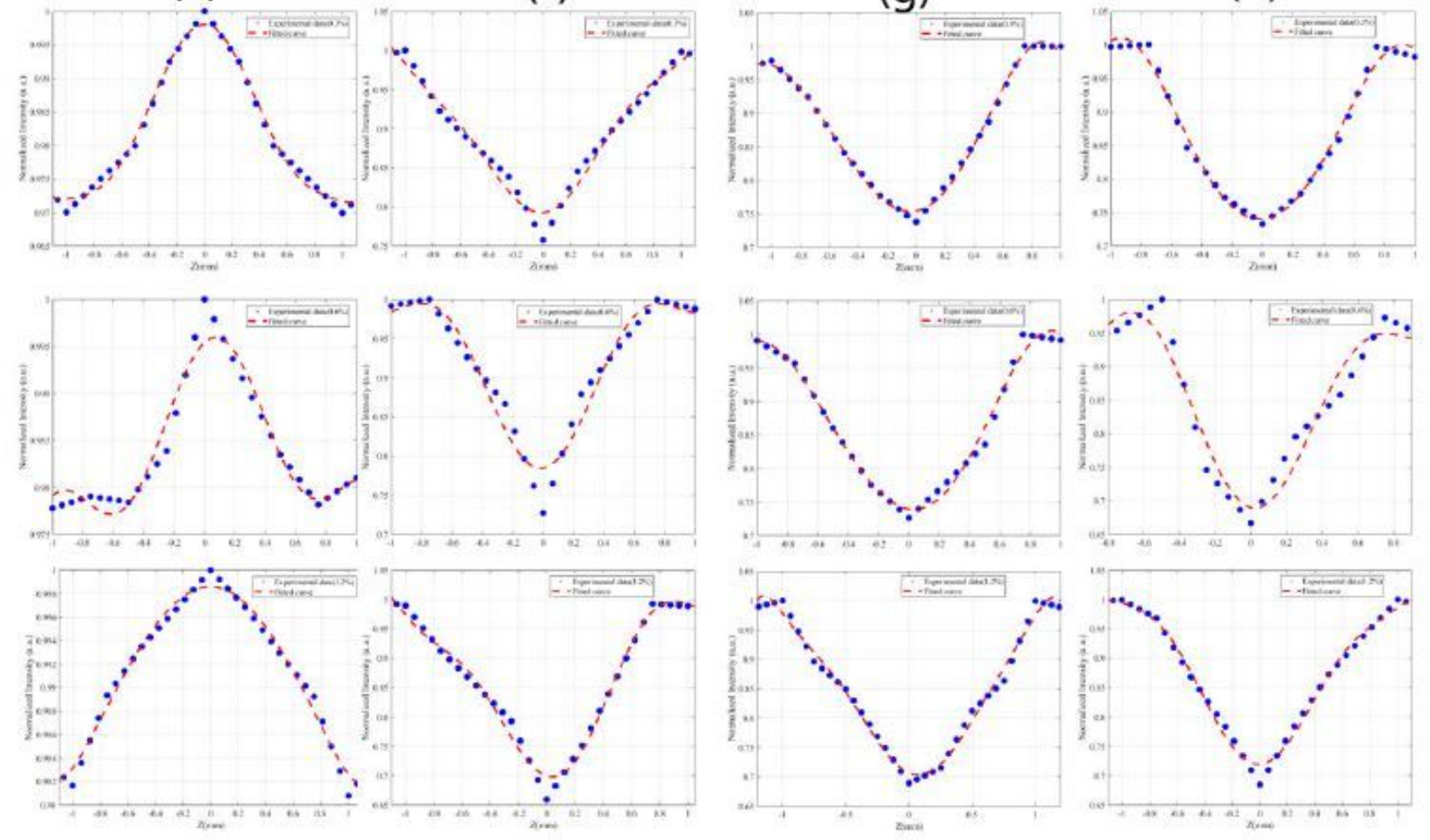

\section{Figure 10}

Nonlinear optical properties of titanium nitride prepared by Ball mill(350 rpm) method. The closed aperture Z-Scan data with different concentration and laser beam power of a: $70 \mathrm{~mW}, \mathrm{~b}: 85 \mathrm{~mW}, \mathrm{c}: 100 \mathrm{~mW}$, and d: $120 \mathrm{~mW}$, the open aperture Z-Scan data with different concentration and laser beam power of e: 70mW, f: $85 \mathrm{~mW}, \mathrm{~g}: 100 \mathrm{~mW}$, and h: 120mW. 
(a)

(b)
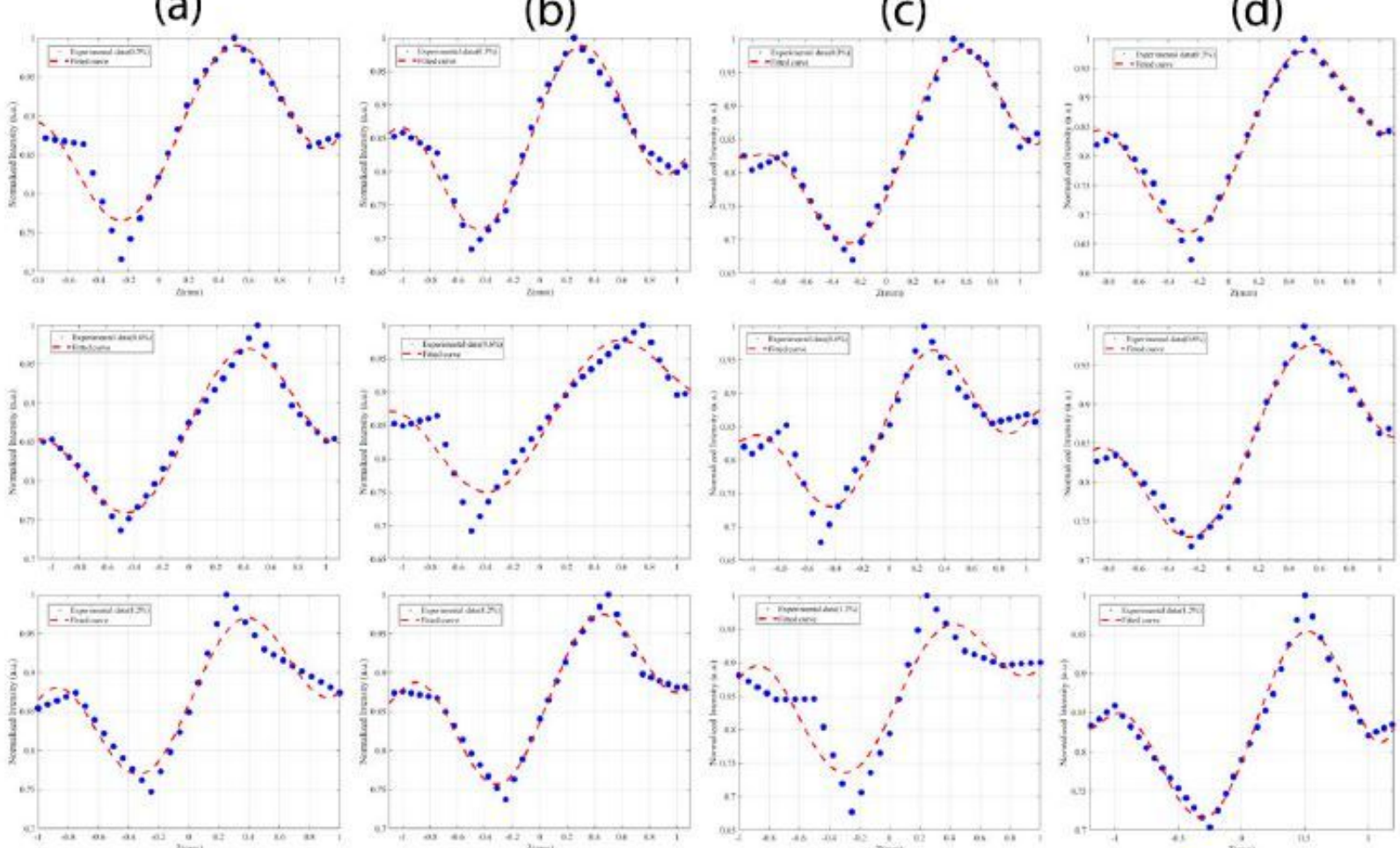

(e)

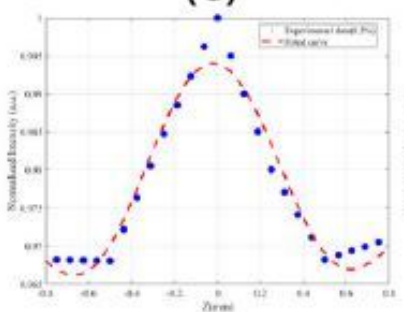

(f)

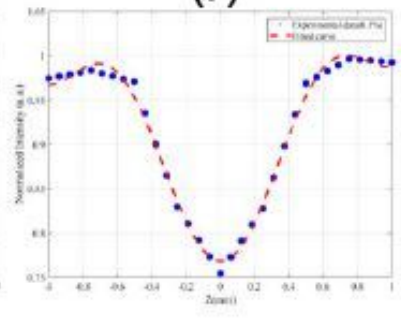

(g)

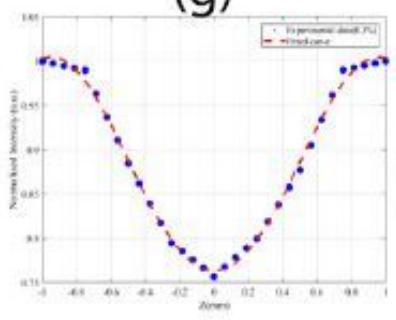

(h)
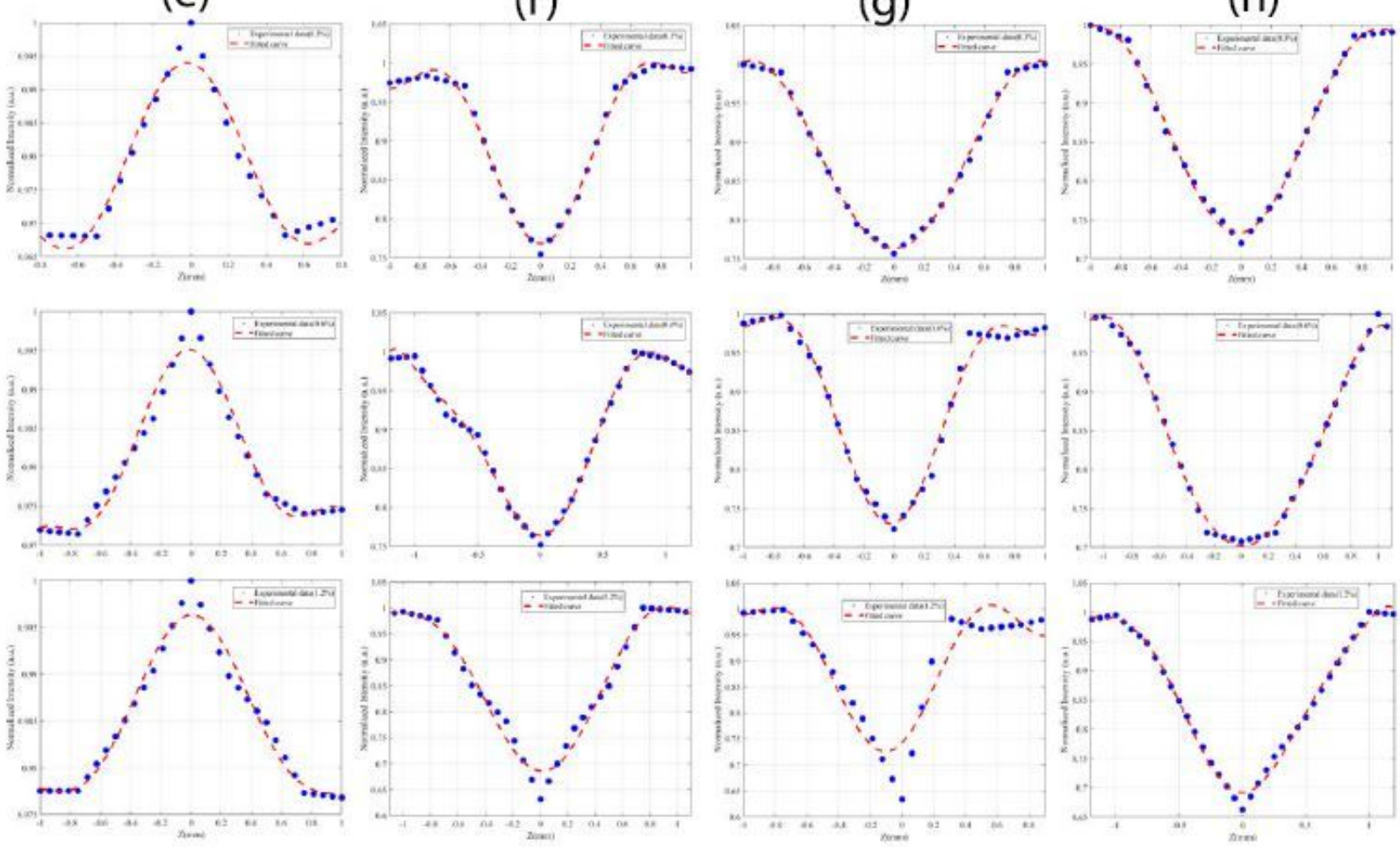

\section{Figure 11}

Nonlinear optical properties of titanium nitride prepared by Ball mill(500 rpm) method. The closed aperture Z-Scan data with different concentration and laser beam power of a: $70 \mathrm{~mW}, \mathrm{~b}: 85 \mathrm{~mW}, \mathrm{c}: 100 \mathrm{~mW}$, and d: $120 \mathrm{~mW}$, the open aperture Z-Scan data with different concentration and laser beam power of e: $70 \mathrm{~mW}$, f: $85 \mathrm{~mW}$, g: $100 \mathrm{~mW}$, and $\mathrm{h}: 120 \mathrm{~mW}$. 
(a)

(b)
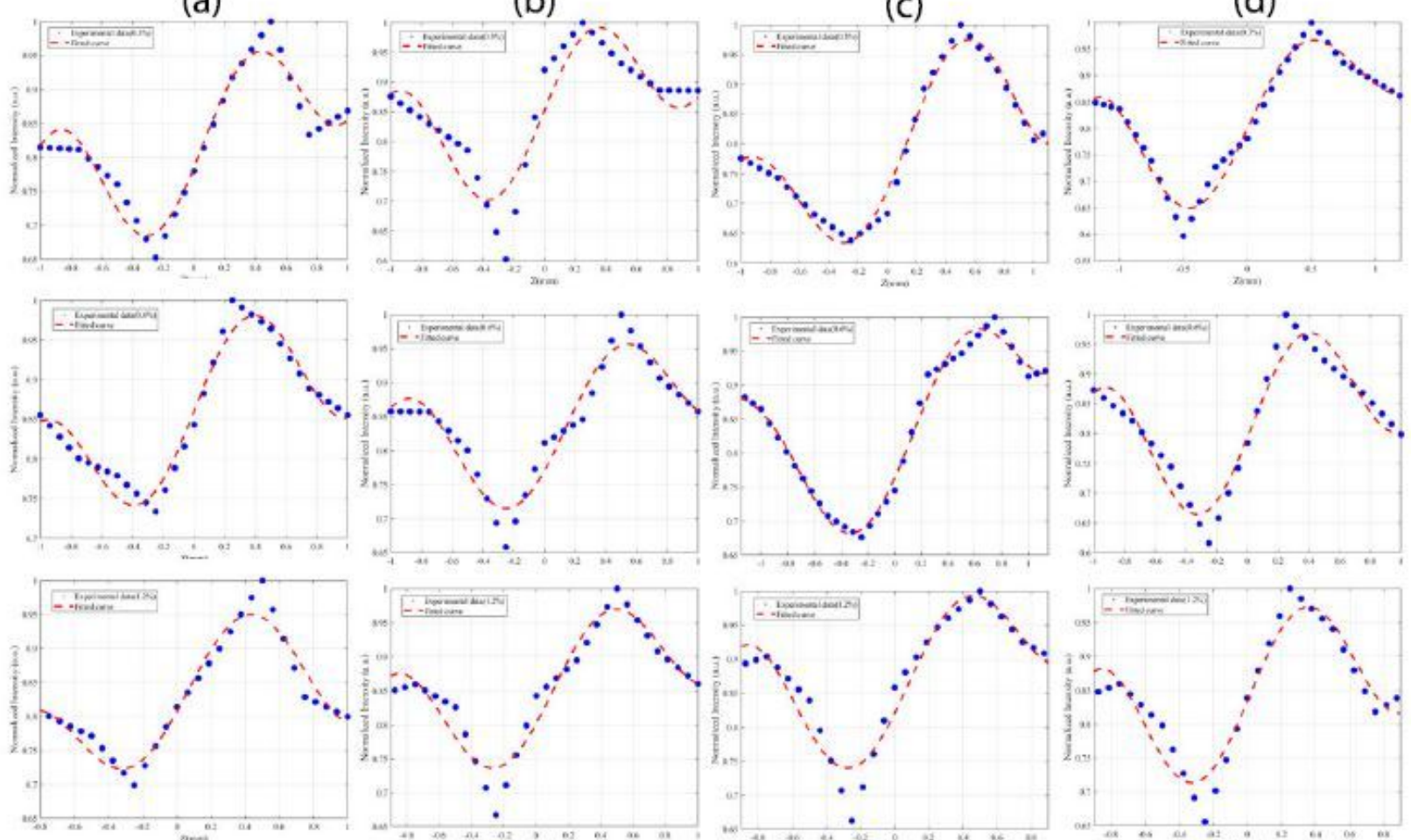

(e)

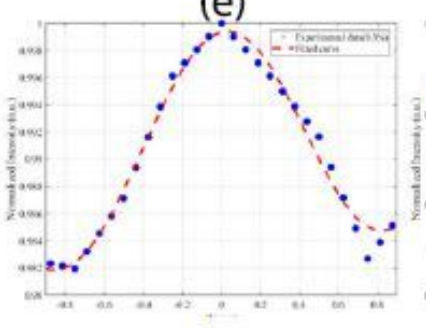

(f)

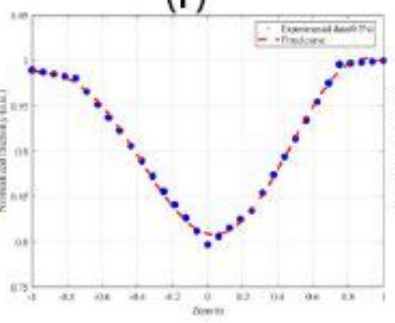

(g)
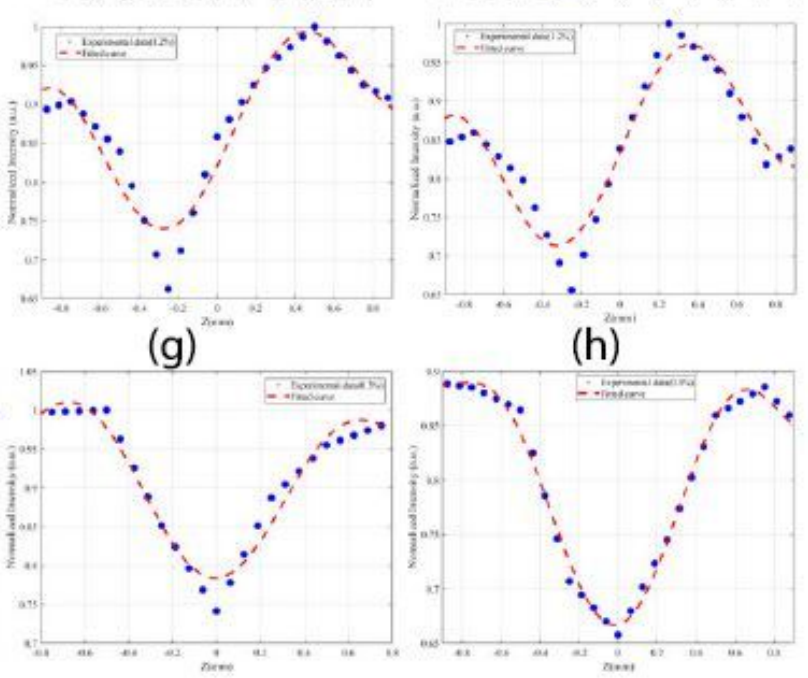

(h)
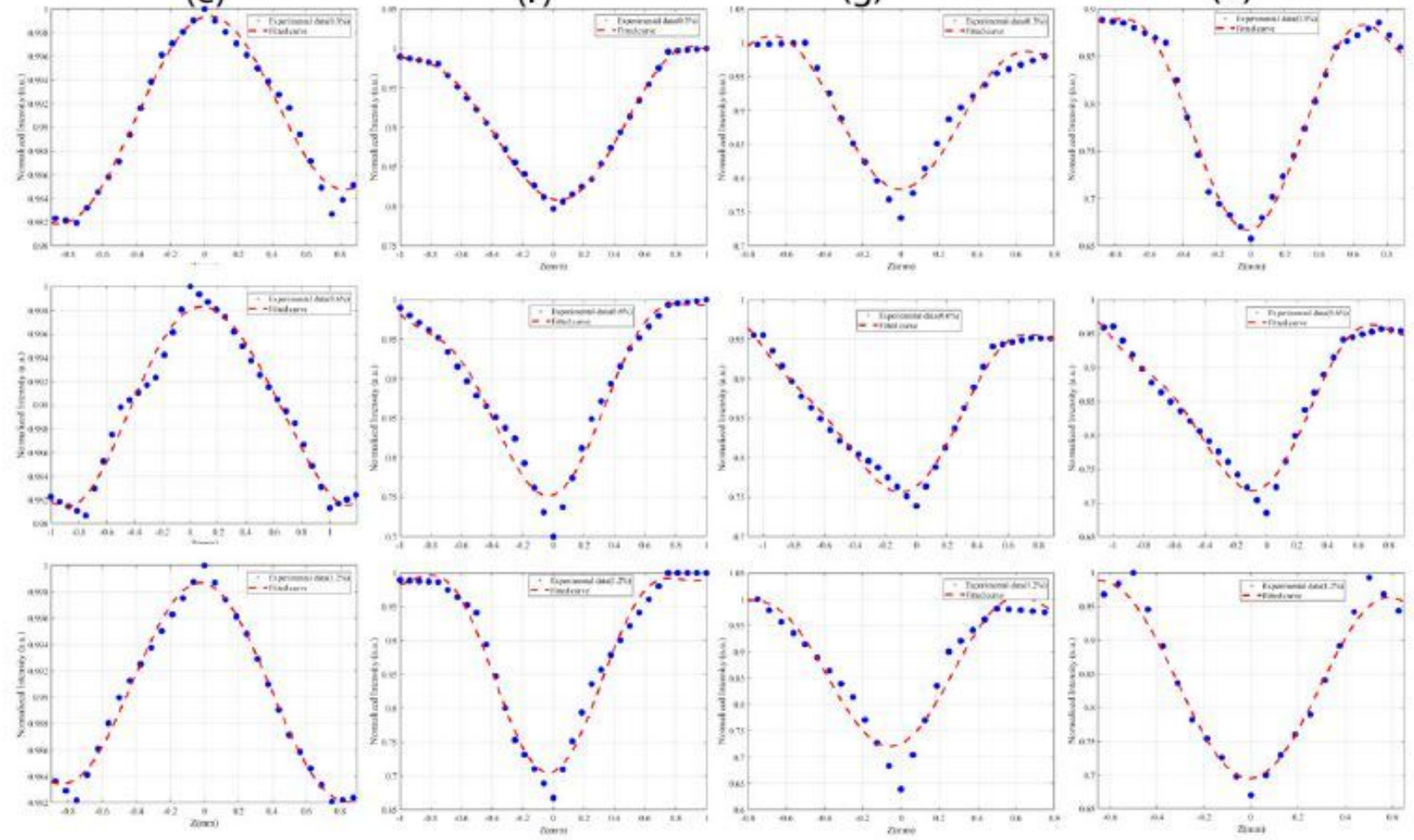

\section{Figure 12}

Nonlinear optical properties of titanium nitride prepared by the co-precipitation method. The closed aperture Z-Scan data with different concentration and laser beam power of a: $70 \mathrm{~mW}, \mathrm{~b}: 85 \mathrm{~mW}, \mathrm{c}: 100 \mathrm{~mW}$, and $\mathrm{d}: 120 \mathrm{~mW}$, the open aperture Z-Scan data with different concentration and laser beam power of e: $70 \mathrm{~mW}$, f: $85 \mathrm{~mW}, \mathrm{~g}: 100 \mathrm{~mW}$, and $\mathrm{h}: 120 \mathrm{~mW}$. 
(a)
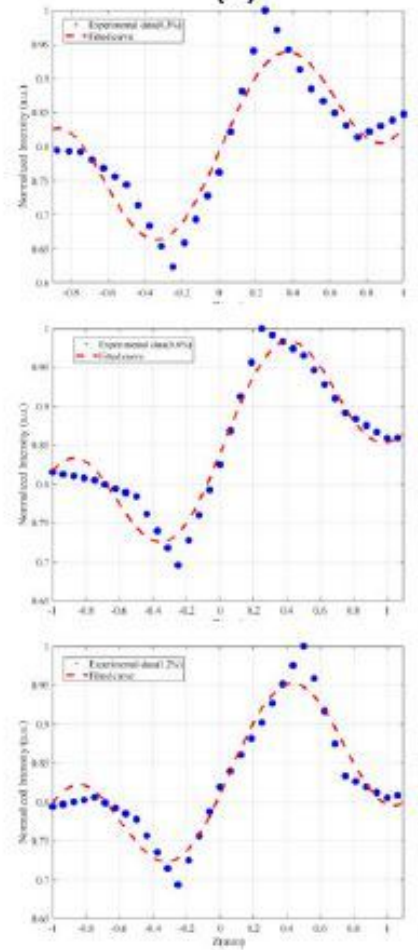

(e)
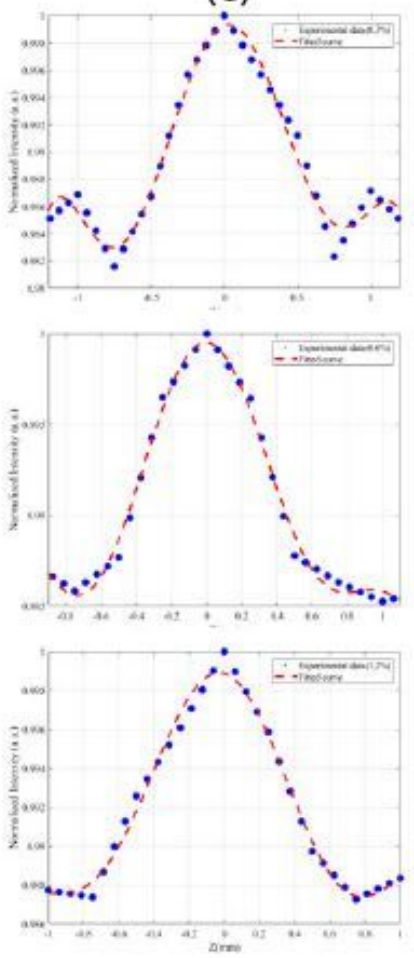

(b)
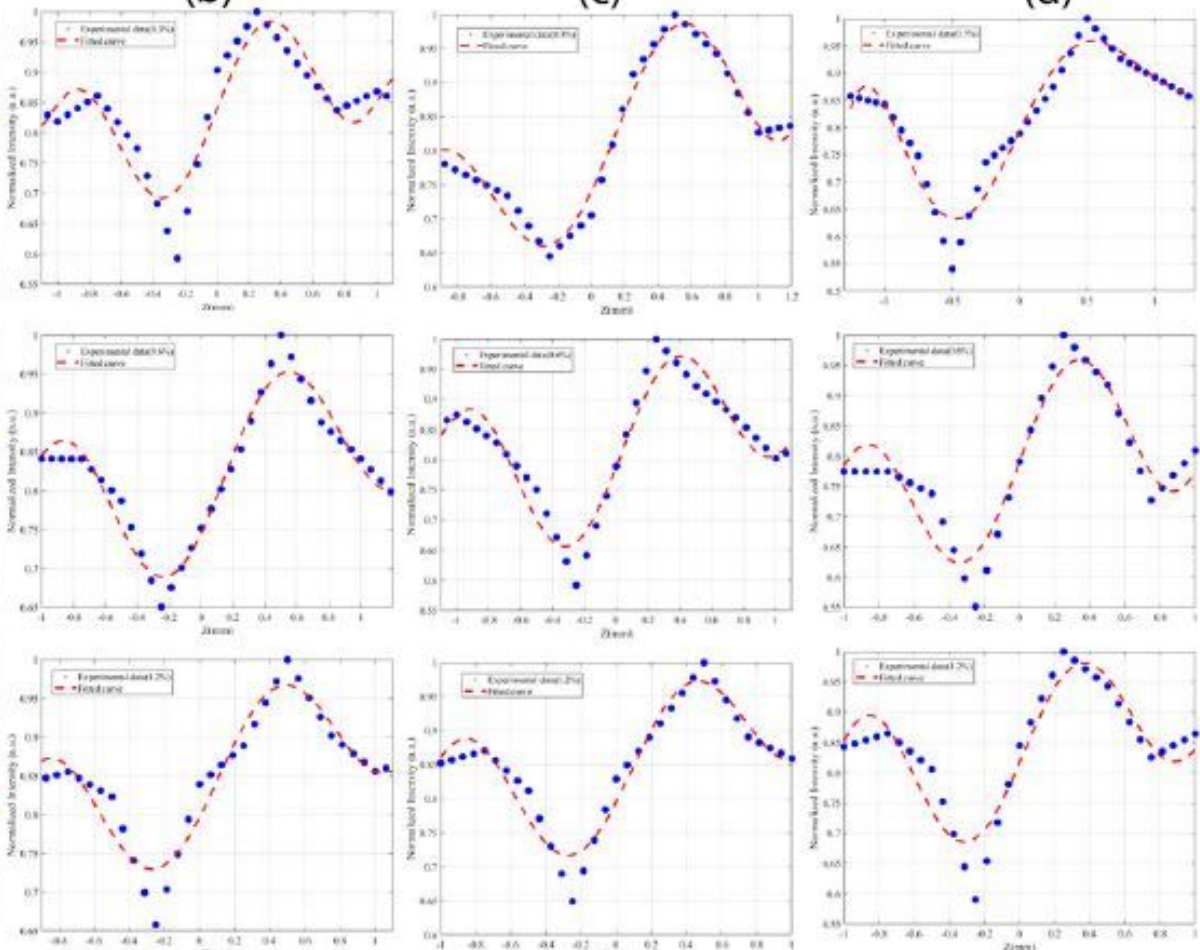

(f)
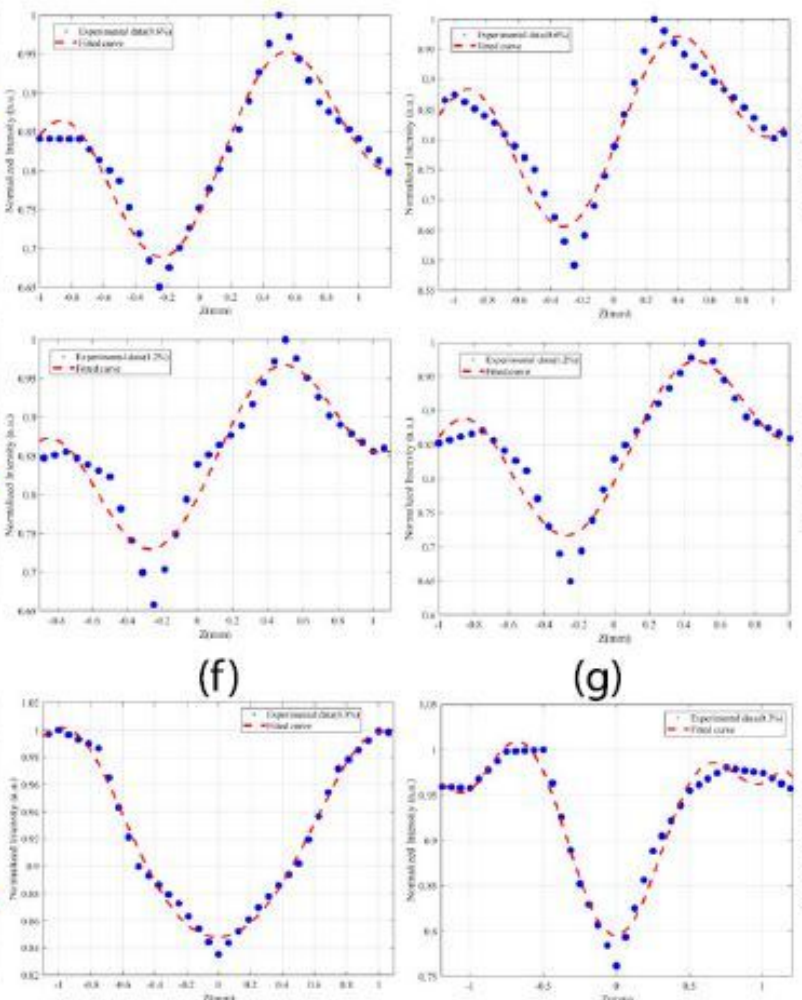

(g)
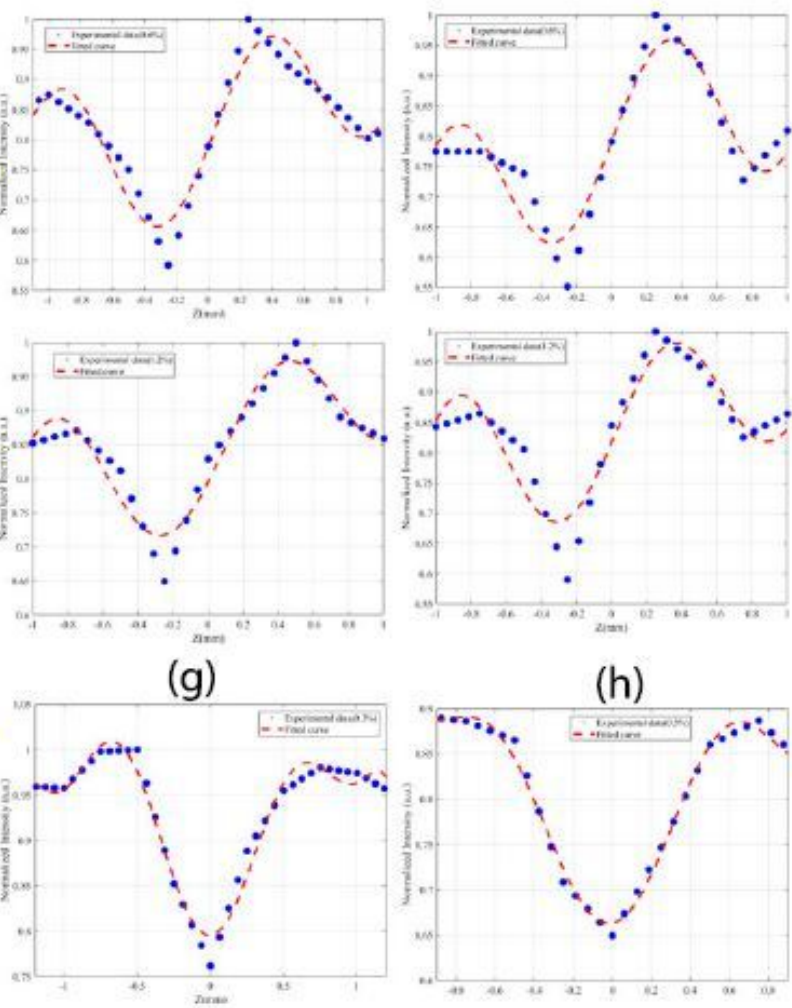

(h)
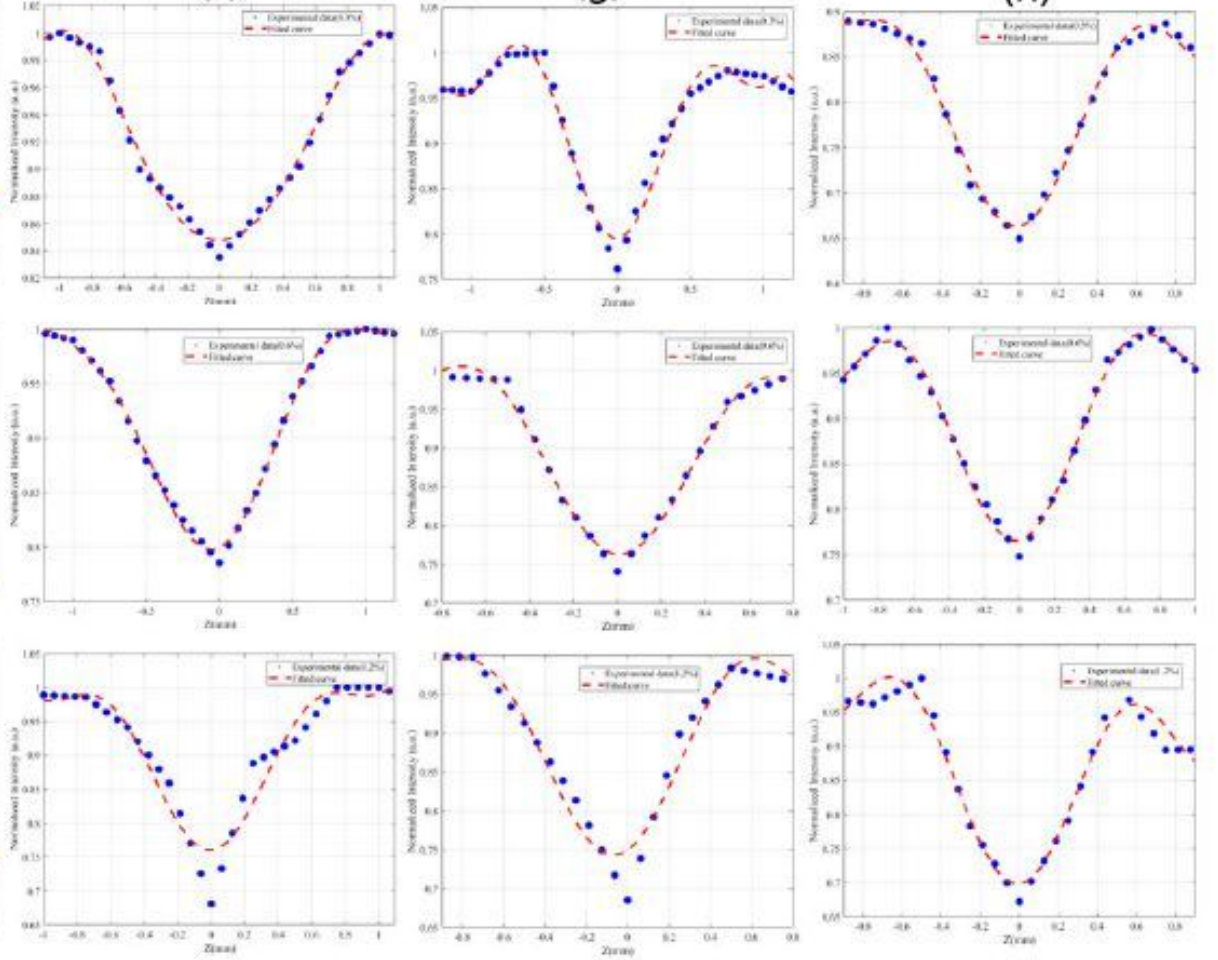

\section{Figure 13}

Nonlinear optical properties of titanium nitride prepared by the sol-gel method. The closed aperture ZScan data with different concentration and laser beam power of a: $70 \mathrm{~mW}, \mathrm{~b}: 85 \mathrm{~mW}, \mathrm{c}: 100 \mathrm{~mW}$, and d: $120 \mathrm{~mW}$, the open aperture Z-Scan data with different concentration and laser beam power of e: $70 \mathrm{~mW}, \mathrm{f}$ : $85 \mathrm{~mW}, \mathrm{~g}: 100 \mathrm{~mW}$, and $\mathrm{h}: 120 \mathrm{~mW}$. 

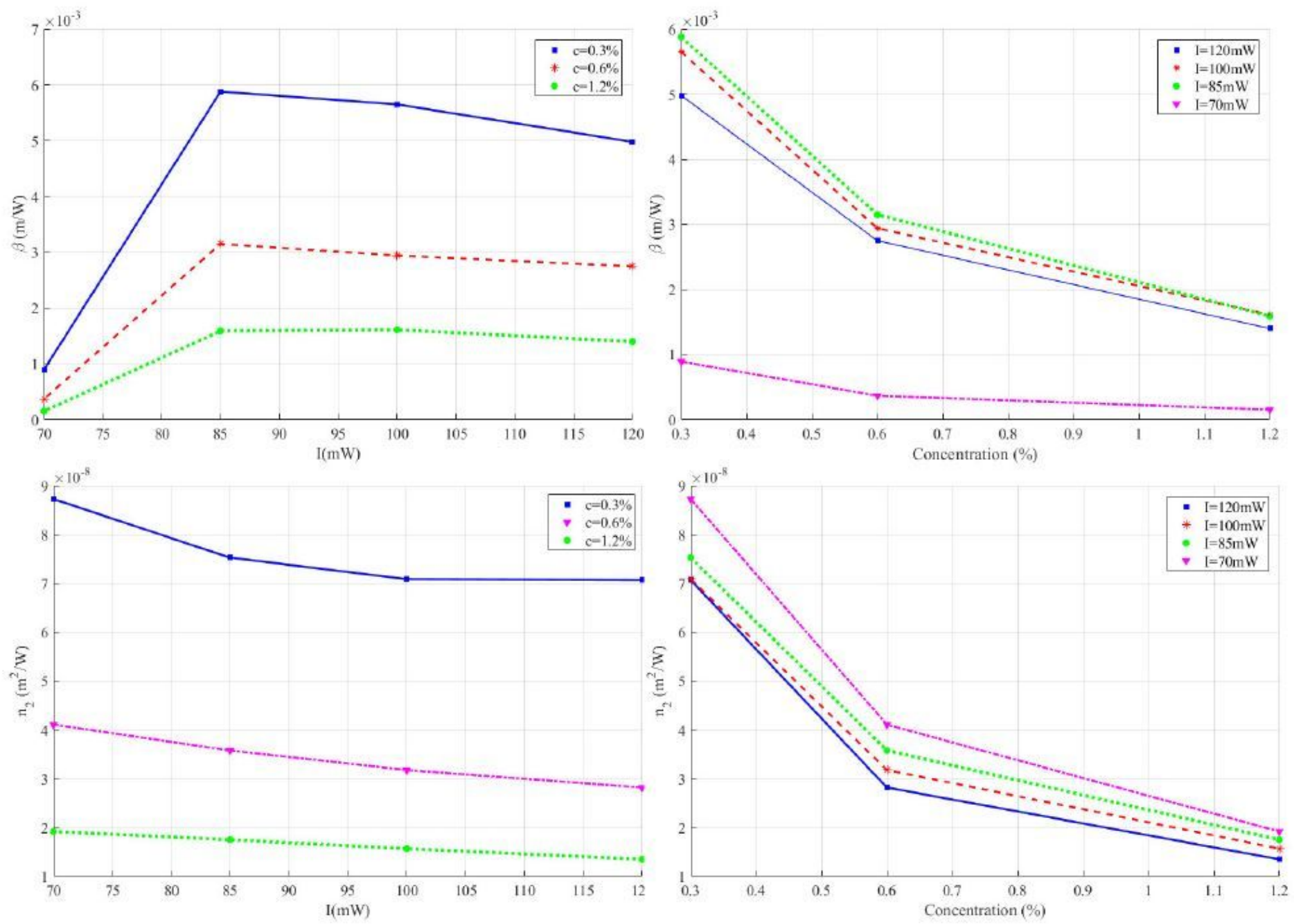

Figure 14

Plot of nonlinear optical properties $(\beta, \mathrm{n} 2)$ against concentration and laser intensity for TiN nanoparticles. 